\title{
Diversidad y patrones de actividad de mamíferos silvestres medianos y grandes en la Cordillera de Talamanca, Costa Rica
}

\author{
Roger González Tenorio' ${ }^{(D)}$, Amy A. Eppert ${ }^{2}$ iD \& Michael S. Mooring ${ }^{2,3}$ iD
}

1. Sistema Nacional de Áreas de Conservación, Amistad Pacífico, 100 m Este y 200 m Sur de la UNED, Pérez Zeledón, Costa Rica; roger.gonzalez@sinac.go.cr

2. Point Loma Nazarene University, Department of Biology, 3900 Lomaland Drive, San Diego, CA 92106, United States; aeppert1998@pointloma.edu, mmooring@pointloma.edu

3. Centro de Educación e Investigación Quetzal, 200 metros al norte del hotel Savegre, San Gerardo de Dota, Costa Rica.

Recibido 5-VII-2021 • Corregido 26-VIII-2021 • Aceptado 5-IX-2021 DOI: https://doi.org/10.22458/urj.v13i2.3621

\begin{abstract}
The diversity and activity patterns of medium and large terrestrial mammals in the Talamanca Mountain Range, Costa Rica". Introduction: The Talamanca Range in the southeast of Costa Rica is a priority region for conservation, but its ecosystems and species are little known. The study of wild mammals can contribute to our understanding of the trophic structure and conservation needs of tropical forests, which are some of the most diverse ecosystems on the planet. Objective: To evaluate the species richness, relative abundance and activity patterns of medium and large mammals in La Amistad National Park and the Quetzal Tres Colinas Biological Corridor. Methods: Continuous monitoring was carried out from July 12, 2018 to April 18, 2021 using 18 phototrapping stations, each consisting of a camera trap and a scent station. Results: Based on a sampling effort of 15 335 camera trap days, we obtained 36667 records in which we detected 27 species of medium and large wild mammals, all of which are in one of the risk categories at the national or international level. The species with the widest distribution and the greatest relative abundance were Sciurus granatensis, Tapirus bairdii, Sylvilagus dicei and Mazama temama. The least abundant species, with the most restricted distribution, were Ateles geoffroyi, Cebus imitator and Microsciurus alfari. Five species were diurnal, six were nocturnal and crepuscular, and 14 species were cathemeral. The greatest species richness was found in the Premontane and Lower Montane zones, while the endemic species were in both of these zones and the Subalpine zone. Eight species had lunarphobia, six lunarphilia and six had no pattern. Conclusion: These areas protect important Premontane to Subalpine populations of medium-size and large terrestrial mammals, many cathemeral or with lunarphobia, and should continue to be monitored.
\end{abstract}

Keywords: Páramo, peat bog, cloud forest, life zone, altitudinal zone.
RESUMEN. Introducción: La Cordillera de Talamanca en el sureste de Costa Rica, es una región prioritaria para la conservación, posee ecosistemas y especies poco conocidas. El estudio de mamíferos silvestres permite comprender la estructura y estado de conservación de los bosques tropicales: uno de los ecosistemas más diversos del planeta. Objetivo: Evaluar la riqueza, abundancia relativa y patrones de actividad de los mamíferos terrestres medianos y grandes en el Parque Internacional de La Amistad y el Corredor Biológico el Quetzal Tres Colinas. Métodos: Entre el 12 de julio del 2018 al 18 de abril del 2021, instalamos 18 estaciones de fototrampeo, cada una conformada por una cámara trampa y un atrayente olfativo. Resultados: Con un esfuerzo de muestreo de 15335 días cámara trampa, obtuvimos 36 667 registros en los cuales detectamos 27 especies de mamíferos silvestres medianos y grandes; todas están en alguna categoría de riesgo nacional o internacional. Sciurus granatensis, Tapirus bairdii, Sylvilagus dicei y Mazama temama mostraron la distribución más amplia y la mayor abundancia relativa. Ateles geoffroyi, Cebus imitator y Microsciurus alfari tuvieron la menor abundancia y la distribución más restringida. Registramos cinco especies diurnas, seis nocturnas-crepusculares y 14 catemerales. Las especies únicas las detectamos en los pisos altitudinales Subalpino, Premontano y Montano bajo, los dos últimos registraron la mayor riqueza. Ocho especies mostraron fobia lunar, seis mostraron filia lunar y seis no mostraron afinidad al brillo lunar. Conclusión: Estas áreas protegen importantes poblaciones premontanas a subalpinas de mamíferos terrestres medianos y grandes, muchos catemerales o con fobia a la luna, y deben seguir siendo monitoreadas.

Palabras clave: Páramo, turbera, bosque nuboso, zona de vida, piso altitudinal. 
Los bosques montañosos menos fragmentados de Costa Rica (C.R.) se ubican entre 1400 y 3 200msnm (Tobler et al., 2006), en la Cordillera de Talamanca, cuya mayor extensión la ocupa el Parque Internacional de La Amistad (PILA) (SINAC, 2019), donde existen ecosistemas aptos para la conservación a largo plazo (Kappelle, 2004; Avalos, 2007). Algunas investigaciones (Carbonell \& Torrealba, 2007; González et al., 2008; Altrichter \& Carbonell, 2013; Mejía, 2014; Chaverri et al., 2016;) han registrado 189 especies de mamíferos silvestres: 23 endémicas de Talamanca, 21 en peligro de extinción y 44 con poblaciones reducidas o amenazadas (Rodríguez et al., 2014).

Los mamíferos silvestres se movilizan entre las Áreas Silvestres Protegidas (ASP) y su entorno (Arias et al., 2008; Céspedes et al., 2008). El Corredor Biológico el Quetzal Tres Colinas (CBQTC) es una estrategia para conservar la biodiversidad fuera de los límites del ASP (Florian et al., 2010; Salom et al., 2010; Villate et al., 2010; Villate y Canet, 2010). La mayor riqueza de especies se da en elevaciones medias y disminuye conforme aumenta la altura (Jaime et al., 2016). Aunque los ecosistemas del PILA (SINAC, 2019) y del CBQTC (Calvo, 2009; Martínez, 2013) albergan especies con poblaciones reducidas o en peligro de extinción (Kappelle, 2004; SINAC, 2019), existe un vacío de información de los mamíferos de las montañas altas (Kappelle, 2004), pues la mayoría de las investigaciones se han realizado en elevaciones medias y bajas (SINAC, 2017).

La riqueza y abundancia de los mamíferos silvestres varía espacial y temporalmente (Walker et al., 2000; Lira \& Briones, 2012), por lo que las especies que existen en el CBQTC y el PILA cambian con respecto a los pisos altitudinales (Ramos et al., 2007), zonas de vida (Williams et al., 2002), estructura y fragmentación del hábitat (Norris et al., 2010). La actividad circadiana de los mamíferos varía con respecto al estrés térmico (Owen, 1998; Oliveira et al., 2010), el día, la noche o el crepúsculo (Van Schaik \& Griffiths, 1996; Kirk, 2006; Bennie et al., 2014), la disponibilidad de alimento (McDonough et al., 1997), el riesgo de depredación (Downes, 2001; Mech \& Cluff, 2011; Russo et al., 2011; Suselbeek et al., 2014), el brillo de la luna (Lizcano \& Cavelier, 2000; Norris et al., 2010; Harmsen et al., 2011; Penteriani et al., 2013), la estación seca o la lluviosa (Santos et al., 2010) y los cambios antropogénicos (Kitchen et al., 2000).

Se requiere generar mayor conocimiento de los mamíferos silvestres (Rodríguez et al., 2002), pues su patrón de actividad, interacción entre especies y ocupación son claves para la conservación (Berger-Tal et al., 2011) y son indicadores de la salud de los ecosistemas, pues sufren variaciones cuando se altera su hábitat (Sanderson \& Trolle, 2005), lo cual afecta la riqueza, grupos funcionales y dominancia (Ahumada et al., 2011). Las actividades humanas en el PILA y el CBQTC afectan el comportamiento de especies o comunidades (Wright et al., 2010) de mamíferos tropicales que utilizan los senderos antrópicos y sus patrones de actividad pueden variar con la actividad humana (Blake et al., 2017), aunque la tendencia de los mamíferos a caminar sobre un sendero es muy variable (Harmsen et al., 2009). Un estudio similar a éste (Ahumada et al., 2013), desarrollado en C.R. en un gradiente altitudinal de 3000 metros, detectó 26 especies varias de ellas con algún riesgo de conservación. Estas investigaciones aportan al conocimiento biológico y fortalecen la gestión de las ASP (Rodríguez et al., 2002) pues son una base para la toma de decisiones de manejo (Vásquez \& Matallana, 2016).

En esta investigación, utilizamos los datos registrados por cámaras trampas para evaluar la riqueza, abundancia relativa, patrones de actividad, distribución espacial y temporal de los mamíferos silvestres medianos y grandes en el CBQTC y el PILA, en un gradiente altitudinal de $2569 m$, en la Cordillera de Talamanca, Costa Rica. 


\section{MATERIALES Y MÉTODOS}

Área de estudio: Realizamos una investigación en un gradiente altitudinal desde $980 \mathrm{msnm}$ en el CBQTC hasta 3 503msnm en el PILA, en el Sur de Costa Rica. El CBQTC tiene una extensión de 1 800,8ha en donde existen paisajes alterados, áreas en regeneración y parches boscosos que brindan conectividad funcional $(93,3 \%)$, estructural $(70 \%)$ y cobertura forestal $(62,5 \%)($ Calvo, 2009$)$ aptas para la conservación de mamíferos. El sendero Tres Colinas-Cerro Kamuk recorre bosques nubosos (Kappelle \& Brown, 2001), turberas y páramos (Kappelle \& Horn, 2005) que forman parte del PILA (Fig. 1), el cual es el ASP terrestre más extensa de C.R. (199 147ha) (SINAC, 2019) cubierta de bosque tropical inalterado (Palminteri et al., 1999). El endemismo y biodiversidad característicos del PILA, le han conferido tres denominaciones internacionales: Reserva de la Biósfera, Sitio de Patrimonio Mundial y Sitio Ramsar (SINAC, 2012).

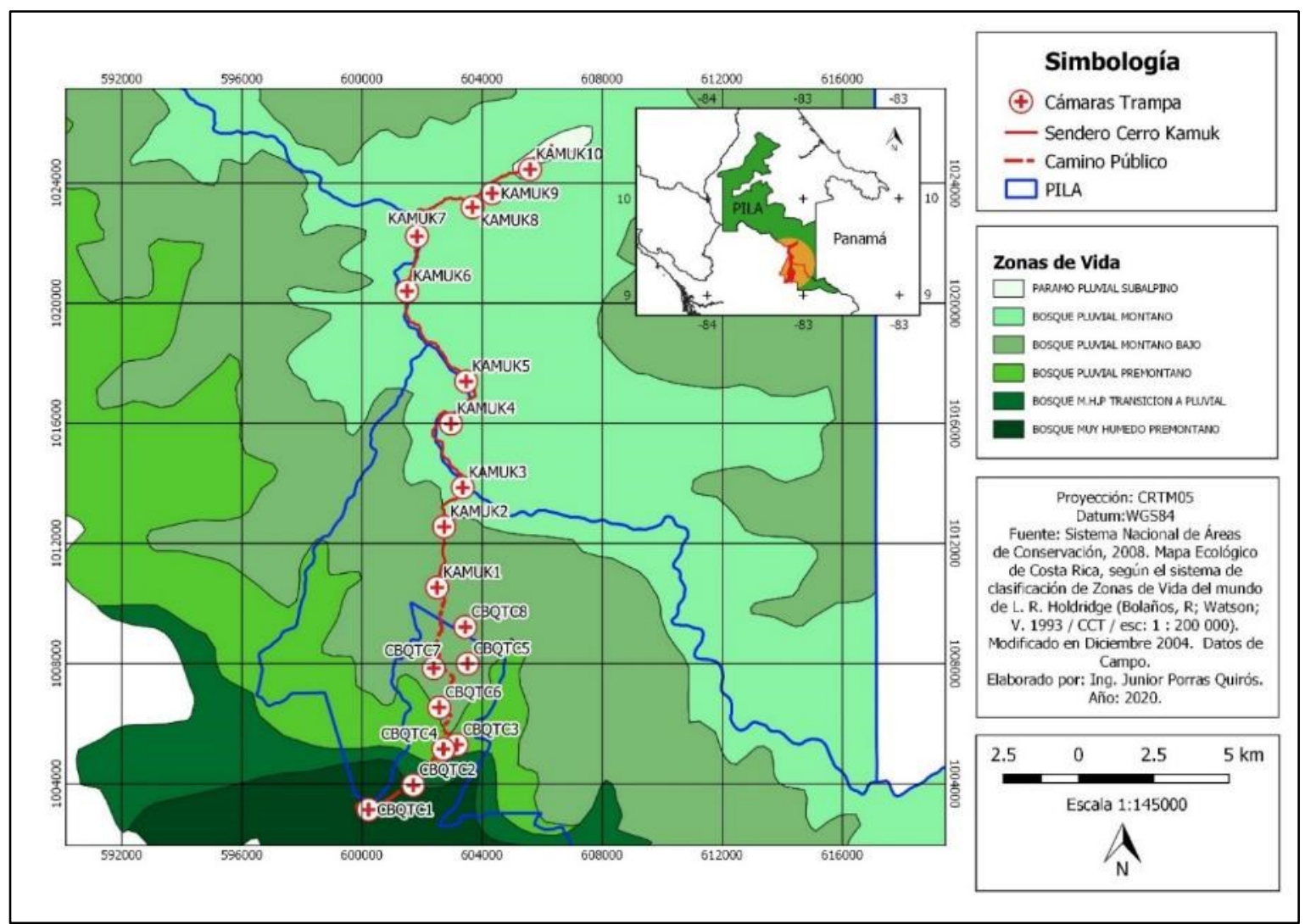

Fig. 1. Ubicación de las estaciones de fototrampeo en el Corredor Biológico el Quetzal Tres Colinas y en el Parque Internacional de La Amistad, Costa Rica

Población de estudio: De las 249 especies de mamíferos silvestres registrados en Costa Rica (Rodríguez et al., 2014), seleccionamos las que se desplazan en el suelo (no voladores ni arborícolas), cuyo tamaño corporal en individuos adultos va de mediano a grande. Determinamos las tallas medianas (peso: $>101 \mathrm{~g}<10 \mathrm{~kg}$ ) y grandes (peso $>10 \mathrm{~kg}$ ) con base en Sánchez et al. (2019) y Arita y Figueroa (1999). Incluimos todos los registros de seres humanos (Homo sapiens) y los mamíferos domésticos: caballos (Equus caballus), vacas (Bos taurus) y perros (Canis familiaris). 
Cámaras trampa: Utilizamos el "fototrampeo" debido a sus ventajas sobre otras técnicas de investigación de mamíferos (Zúñiga \& Jiménez, 2010; Janecka et al., 2011; O’Connell et al., 2011; Vila et al., 2016; Hurtado et al., 2018) y seguimos el procedimiento y ajustes para C.R. realizados por Hurtado y Soto (2017). Utilizamos 18 cámaras trampa (Bushnell Trophy Cam Essential E3 Brown) colocadas dentro de una caja de acero con candado de seguridad y atadas a un árbol robusto mediante un cable antirrobo Masterlock Python (Mooring et al., 2020). El flash infrarrojo de las cámaras evitó el destello blanco que podría asustar a los animales en condiciones de poca luz (Séquin et al., 2003; Gibeau \& McTavish, 2009; Meek et al., 2014; Meek et al., 2016; Srbek et al., 2018). Instalamos las cámaras a $50 \mathrm{~cm}$ sobre el suelo, orientadas en el mismo sentido del paso de animales para aumentar el tiempo dentro del campo de visión de cada cámara (Mooring et al., 2020). Ajustamos el sensor infrarrojo en modo normal y las programamos para activarse automáticamente cada vez que detectaron calor corporal y movimiento, en intervalos de cada tres segundos, tomando tres fotografías continuas (en calidad 4K), que se almacenaron en tarjetas SD rotuladas con el número de cada cámara trampa. Cada dos meses recolectamos las tarjetas, revizamos el nivel de carga de las baterías, la configuración de cada cámara y ajustamos la hora y fecha correctas para conocer el patrón de actividad de las especies a través de los metadatos Exchangeable Image File Format (EXIF) de fecha y hora (Griffiths \& Van Schaik, 1993; Gómez et al., 2005) grabados en cada fotografía.

Estaciones de olor: Colocamos un atrayente olfativo (Moreno, 2000; Polisar et al. 2014) a dos metros frente a cada cámara trampa. El atrayente consistió en un trozo $(5 \mathrm{~cm})$ de esponja comercial impregnada con colonia para hombre (Obsession de Calvin Klein $\mathrm{CK}^{\circledR}$ ) colocada dentro de $6 \mathrm{~cm}$ de tubo de vinilo transparente, el cual atamos al extremo de un tubo de PVC incrustado verticalmente en el suelo (Mooring et al., 2020). Aunque el atrayente olfativo mantuvo su efecto por más de tres meses (Maxwell, 2018), durante nuestras giras colocamos más perfume rociando cuatro veces sobre la esponja (Mooring et al., 2020). Esto aumentó la calidad de las fotografías y ayudó en la identificación de especies y de individuos (Weaver et al., 2005; Barea et al., 2007; Thorn et al., 2009; Randel \& Peace, 2010; Maffei et al., 2011; García, 2012; Braczkowski \& Watson, 2013; Noss et al., 2013). La estación de olor atrajo sólo los mamíferos que ya estaban cerca (Maxwell, 2018) pues el olor se detecta a máximo 20 metros (Jinn, 2019) y no afectó la cantidad de fotografías, distancias de movimiento, inmigración o emigración, actividad temporal ni abundancia (Gerber et al., 2012; Braczkowski et al., 2016).

Diseño de muestreo: Para reflejar los ecosistemas del PILA y el CBQTC, combinamos el muestreo sistemático y selectivo (Hurtado \& Soto, 2017). Cada estación de fototrampeo se instaló considerando que entre ellas existiera una distancia mínima de 2 km (Rovero \& Marshall, 2009; Parsons et al., 2017) y que hubiera al menos dos estaciones por zona de vida, así como rastros de mamíferos silvestres (Karanth et al., 2006) y árboles grandes en donde colocar las cámaras trampa. Utilizamos el programa Quantum Giz 2,8 (QGIS, 2020) y el Atlas Digital de C.R. (Ortiz, 2014) para trazar una cuadrícula de $1 \times 1 \mathrm{~km}$ sobre el sitio de estudio. Instalamos estaciones de fototrampeo en el borde de senderos, caminos, servidumbres y carriles. Tramitamos el permiso de investigación RSINAC-PNI-ACLAP-016-2021 y en el CBQTC obtuvimos la autorización de los propietarios. Las estaciones de fototrampeo permanecieron en un mismo sitio el cual georreferenciamos mediante un GPS Garmin 64s (Apéndice digital, Tabla 1). Prolongamos el tiempo de muestreo durante las 24 horas desde el 12 de julio del 2018 al 18 de abril del 2021 (1 012 días), lo cual brindó un esfuerzo de muestreo de 15335 días cámaras trampa, pues el tiempo necesario para investigar mediante fototrampeo es inversamente proporcional al número de cámaras instaladas (Hurtado et al., 2018). 
Análisis de datos: Almacenamos las fotografías en una computadora y las revisamos para detectar la presencia de mamíferos e identificarlos taxonómicamente. Hicimos una carpeta para cada cámara trampa, dentro de las cuales creamos subcarpetas por especie y dentro de éstas hicimos subcarpetas con el número de individuos observados en cada fotografía. El programa Renamer (Kozlov, 2018) nos permitió renombrar cada fotografía con sus metadatos EXIF (año-mesdía, hora-minuto-segundo). Renamer generó un archivo de texto (.txt) que analizamos mediante CameraSweet (Sanderson \& Harris, 2013) y obtuvimos resultados de riqueza, abundancia relativa, patrones de actividad circadiana y esfuerzo de muestreo. Analizamos sólo los eventos independientes separados por al menos 30 minutos de cualquier otro registro fotográfico de la misma especie y en la misma cámara trampa (Ridout \& Linkie, 2009; Si et al., 2014; Van Berkel, 2014; Anile \& Devillard, 2016; Sollmann, 2018). Exportamos la base de datos creada por CameraSweet y la guardamos como un archivo de Excel (.csv) que analizamos mediante guiones escritos en el lenguaje de programación $\mathrm{R}$ ( $\mathrm{R}$ Core Team, 2019). Seleccionamos el programa con base en Young et al. (2018).

Riqueza de especies: Identificamos todas las especies de mamíferos y sus gremios tróficos con base en Mora (2000), Carrillo et al. (2002) y Wainwright (2007) y los clasificamos como herbívoros, insectívoros, frugívoros, carnívoros y omnívoros (Ceballos y Navarro, 1991). Mediante el programa R (R Core Team, 2019) y su función "Specaccum" del paquete "Vegan", construimos una curva de acumulación de especies. Utilizamos los días acumulados de las cámaras trampa siguiendo el método aleatorio: cada día que las cámaras estuvieron activas se incluyeron en la curva en orden aleatorio (Oksanen et al., 2019). Este orden fue aleatorizado 1000 veces y se calcularon intervalos de confianza del 95\% (Rovero et al., 2014).

Consultamos el estado de conservación de cada especie según la Lista Roja de la Unión Internacional para la Conservación de la Naturaleza (UICN, 2020), la Convención sobre el Comercio Internacional de Especies Amenazadas de Fauna y Flora (CITES) y la lista oficial de especies en peligro de extinción y con poblaciones reducidas y amenazadas del Ministerio de Ambiente y Energía (MINAE) (SINAC, 2017). Asignamos un valor a cada especie de acuerdo con su clasificación: en peligro (5), vulnerable (4), casi amenazado (3), preocupación menor (2) y datos insuficientes (1) (UICN, 2012); Apéndices CITES I (3), II (2) y III (1) (PNUMA-CMCM, 2014); especies con Poblaciones Reducidas (1) y especies en peligro de extinción (2) (MINAE). Sumamos los tres valores (CITES, UICN y MINAE) y categorizamos las especies de acuerdo a su estado de conservación.

Abundancia relativa: La cual calculamos mediante el Índice de Abundancia Relativa (IAR) como la frecuencia con que las cámaras trampa registraron cada especie en todo el sitio y en cada piso altitudinal y zona de vida. Calculamos el IAR mediante la fórmula [(número de observaciones independientes) / (número de días que la cámara estuvo activa)] * 1000 (Rovero \& Marshall, 2009; Lira \& Briones, 2011; Monroy et al., 2011; Cortés \& Briones, 2014; Lira et al., 2014). Utilizamos el IAR debido a su efectividad (Maffei et al., 2002; Azuara, 2005; Jenks et al., 2011; Hernández et al., 2015) y porque la información para calcular la densidad de los mamíferos neotropicales (Negroes et al., 2010; Morgan et al., 2018) es costosa y difícil de obtener (Lira \& Briones, 2012).

Uso del espacio: Mediante el fototrampeo detectamos diversidad y patrones de actividad (Cuellar et al., 2006; Soria \& Monroy, 2015) en cuatro pisos altitudinales y seis zonas de vida (Holdridge, 1987): Bosque Muy húmedo Premontano (BmH-P), Bosque Muy húmedo Premontano Transición a Pluvial (BmHP-TP), Bosque Pluvial Premontano (BP-P), Bosque Pluvial Montano Bajo (BP-MB), Bosque Pluvial Montano (BP-M) y Bosque Pluvial Subalpino(BP-S). Distribuimos las estaciones de fototrampeo de la siguiente forma: seis estaciones en el piso altitudinal Premontano 
(dos en cada zona de vida), cuatro en el Montano Bajo, seis en el Montano y dos estaciones en el piso Subaplino. Analizamos el uso del espacio de las especies por modalidad de conservación mediante la instalación de diez estaciones en el PILA y ocho en el CBQTC (Apéndice digital, TABLA 1).

Patrones de actividad: Determinamos los patrones de actividad circadiana y actividad lunar durante la estación seca y la lluviosa, considerando que las actividades de los mamíferos se asocian con los períodos de luz (día), oscuridad (noche) (Bennie et al., 2014) y estados intermedios (crepusculares o catemerales) (Van Schaik \& Griffiths, 1996; Kirk, 2006). Consideramos especies nocturnas aquellas que detectamos una hora después de la puesta del sol y una hora antes de la salida del sol del día siguiente; las especies registradas entre la salida y la puesta del sol las clasificamos como diurnas. Si el evento ocurrió una hora antes del amanecer o una hora después del atardecer la consideramos una especie crepuscular. Consideramos especies catemerales las que estuvieron activas durante el día, la noche y el crepúsculo (O’Brien et al., 2003).

El momento del amanecer y atardecer en el trópico varía durante el año (Nouvellet et al., 2012) por lo cual analizamos los patrones de actividad circadiana utilizando la hora del sol mediante la función "sunTime" de "Overlap", que funciona con el tiempo en radianes (06:00 horas=p/2 y 18:00 horas $=3 p / 2$ ) (Meredith \& Ridout, 2018a, 2018b). Caracterizamos el patrón de actividad lunar de las especies nocturnas utilizando la función "getMoonlllumination" del paquete "suncalc" (Agafonkin \& Thieurmel, 2018), para cada evento obtuvimos la fase lunar con valores de 0 (luna nueva) 0,25 (cuarto creciente), 0,5 (luna llena) y 0,75 (cuarto menguante), los cuales multiplicamos por $2 \pi$ y escalamos linealmente en radianes para cada ciclo lunar.

Comparamos la actividad de los mamíferos durante la estación seca (noviembre a abril) y la estación lluviosa (mayo a noviembre) (Kappelle, 1996). El Sendero Tres Colinas-Cerro Kamuk estuvo abierto a la visitación turística del 1 de noviembre al 30 de abril de cada año (estación seca) y contó con un estudio de capacidad de carga (Brenes et al., 2004) que reguló el ingreso diario a máximo nueve personas (SINAC, 2019). Mediante la bitácora para el registro de visitantes del SINAC-ACLAPPILA calculamos la cantidad de turistas que ingresaron durante la época seca 2019-2021.

Para determinar si la presencia de humanos y mamíferos domésticos alteró el patrón de actividad (P) de las especies silvestres, analizamos la actividad circadiana y el coeficiente de superposición $(\boldsymbol{\Delta})$ entre todas las especies. Estimamos el $\boldsymbol{\Delta}$ con valores que van desde 0 (sin superposición) a 1 (superposición completa) (Ridout \& Linkie, 2009) lo cual pudo indicar competencia o depredación (Carver et al., 2011; Linkie \& Ridout, 2011; Carter et al., 2012; Kamler et al., 2012; Ramesh et al., 2012; Ross et al., 2013). Realizamos tres pruebas complementarias al $\boldsymbol{\Delta}$ pues las estadísticas asociadas con los datos cíclicos no están tan desarrolladas como las estadísticas tradicionales. La primera prueba complementaria fue "Watson's Two Sample U² Test with Ties" (Zar, 1999) y los valores de nivel de significancia (P) calculados a través de una aproximación de "Chicuadrado" de la distribución de $U^{2}$ (Tiku, 1965). La segunda prueba fue " $W_{r}$ uniform scores statistic", con valores P calculados de acuerdo con Fisher (1993). La tercera prueba fue "Fisher's Exact Test" (Upton, 1992) con valores de $\mathbf{P}$ calculados usando 1000 réplicas de Monte Carlo.

Para determinar el valor de patrón de actividad en cada especie, ajustamos de forma no paramétrica como funciones de densidad del núcleo con el paquete "Overlap" y utilizando los parámetros de ancho de banda predeterminados (Meredith \& Ridout, 2018a, 2018b); siguiendo el supuesto de que todas las especies tienen la misma probabilidad de ser detectadas en cualquier período de su actividad (Linkie \& Ridout, 2011). Para no sobreestimar el patrón de actividad y el $\boldsymbol{\Delta}$ utilizamos un tamaño mínimo de 20 eventos independientes (n) (Lynam et al., 2013; Rowcliffe et al., 2014; Tan et al., 2018). 


\section{RESULTADOS}

Riqueza y estado de conservación: Con un esfuerzo de muestreo de 15335 días cámara trampa proveniente de 18 estaciones de fototrampeo, obtuvimos 36667 registros en los que detectamos 27 especies silvestres de mamíferos medianos y grandes, de las cuales 25 las registramos en el CBQTC y 24 en el PILA. El orden mejor representado fue Carnívora, con cinco familias y 12 especies, seguido por Rodentia con cuatro familias y cinco especies. Detectamos ocho especies carnívoras, siete herbívoras, siete omnívoras, tres frugívoras y dos insectívoras, de las cuales, la mayor abundancia relativa la detectamos en Sciurus granatensis, Tapirus bairdii, Sylvilagus dicei y Mazama temama (herbívoras) y Cuniculus paca (frugívora). De acuerdo con la lista roja de la UICN, registramos dos especies en peligro, cuatro vulnerables, dos casi amenazadas, 18 con preocupación menor y una con datos insuficientes. Ocho especies están en el Apéndice I y II en el Apéndice II de CITES. Nueve especies están en peligro de extinción y cinco tienen poblaciones reducidas según la lista del MINAE (Apéndice digital, Tabla 2). En los primeros 19 días de muestreo detectamos trece especies y requerimos 991 días más para detectar las demás (Apéndice digital, Tabla 3). A través del programa $R$ ( $R$ Core Team, 2019), generamos la curva de acumulación de especies (Fig. 2), la cual tiende a ajustarse a la asíntota. La zona de color gris representa un intervalo de confianza del $95 \%$.

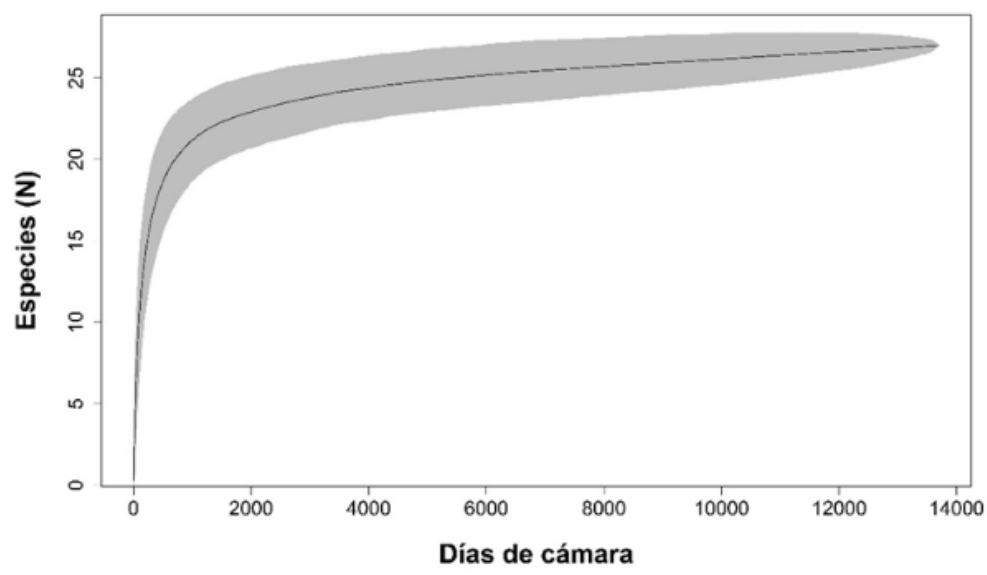

Fig. 2. Curva de acumulación de las especies detectadas en el Corredor Biológico el Quetzal Tres Colinas y el Parque Internacional de La Amistad, Costa Rica

Abundancia relativa: Para todo el sitio, las especies más abundantes fueron $S$. granatensis (IAR=133,41; $\mathrm{n}=1838)$, T. bairdii $(\mathrm{IAR}=53,42 ; \mathrm{N}=736)$, S. dicei $(\mathrm{IAR}=25,91 ; \mathrm{N}=357), \mathrm{M}$. temama $(\operatorname{IAR}=20,90 ; \mathrm{N}=288), C$. paca $(\operatorname{IAR}=20,32 ; \mathrm{N}=280)$, P. onca $(\operatorname{IAR}=11,40 ; \mathrm{N}=157), P$. concolor (IAR=10,67; $\mathrm{N}=147)$ y $D$. marsupialis (IAR=9,15; $\mathrm{N}=126)$. Las especies menos abundantes fueron $C$. latrans $(\mathrm{IAR}=0,87 ; \mathrm{N}=12), M$. frenata $(\mathrm{IAR}=0,65 ; \mathrm{N}=9), H$. yagouaroundi $(\mathrm{IAR}=0,36 ; \mathrm{N}=5), T$. pecari $(\mathrm{IAR}=0,36 ; \mathrm{N}=5)$, A. geoffroyi, $C$. imitator y $M$. alfari (IAR=0,07; $\mathrm{N}=1$ ) (Tabla 1$)$.

En la zona de vida BmH-P, las especies más abundantes fueron $D$. novemcinctus (IAR=74,21), $P$. oposum $(\operatorname{IAR}=47,8)$ y $S$. granatensis (IAR=41,51); las menos abundantes fueron $S$. dicei, $H$. yagouaroundi y $C$. imitator (IAR=1,26). En el BmHP-TP, las especies más abundantes fueron $S$. granatensis (IAR=98,56), M. temama $(I A R=41,07)$ y $N$. narica $(I A R=22,59)$; las menos abundantes fueron $T$. pecari $(I A R=2,05), D$. punctata $(I A R=1,03)$ y $C$. latrans $(I A R=1,03)$. En el BP-P las especies más abundantes fueron $S$. granatensis $(I A R=219,40)$, $T$. bairdii $(\operatorname{IAR}=62,56)$ y $C$. paca $(\operatorname{IAR}=54,40)$; las menos abundantes fueron $H$. yagouaroundi, L. tigrinus y L. pardalis (IAR=0,91) (Tabla 1). 
En el BP-MB las especies más abundantes fueron S. granatensis (IAR=277,40), C. paca $(\operatorname{IAR}=65,20)$ y $S$. dicei $(I A R=50,50)$; las especies menos abundantes fueron $L$. pardalis $(I A R=0,64), A$. geoffroyi $(I A R=0,32)$ y $M$. frenata (IAR=0,32). En el BP-M las especies más abundantes fueron $S$. granatensis $(I A R=77,03)$ y $T$. bairdii $(I A R=67,13)$; y las menos abundantes fueron $D$. marsupialis $(I A R=0,13)$ y $T$. pecari $(I A R=0,13)$. En el BP-S las especies más abundantes fueron $T$. bairdii $(I A R=56,58)$, $S$. granatensis (IAR=40,12) y $M$. temama (IAR=13,37); y las menos abundantes fueron N. narica $(\mathrm{IAR}=1,03)$, P. tajacu $(\mathrm{IAR}=1,03)$ y M. alfari $(\mathrm{IAR}=0,51)$ (Tabla 1).

TABLA 1

Riqueza y abundancia relativa de los mamíferos silvestres medianos y grandes detectados en el Corredor Biológico el Quetzal Tres Colinas y el Parque Internacional de La Amistad, Costa Rica

\begin{tabular}{|c|c|c|c|c|c|c|c|c|}
\hline Especie & $\mathrm{n}^{*}$ & $\begin{array}{c}* * \text { IAR total } \\
\text { del sitio }\end{array}$ & $\begin{array}{c}\text { IAR } \\
\text { BmH-P }\end{array}$ & $\begin{array}{l}\text { IAR BmHP- } \\
\text { TP }\end{array}$ & $\begin{array}{l}\text { IAR } \\
\text { BP-P }\end{array}$ & $\begin{array}{c}\text { IAR } \\
\text { BP-MB }\end{array}$ & $\begin{array}{c}\text { IAR } \\
\text { BP-M }\end{array}$ & $\begin{array}{l}\text { IAR } \\
\text { BP-S }\end{array}$ \\
\hline Sciurus granatensis & 1838 & 133,41 & 41,51 & 98,56 & 219,40 & 277,40 & 77,03 & 40,12 \\
\hline Tapirus bairdii & 736 & 53,42 & 13,84 & 16,43 & 62,56 & 37,71 & 67,13 & 56,58 \\
\hline Sylvilagus dicei & 357 & 25,91 & 1,26 & 5,13 & & 50,50 & 24,82 & 8,74 \\
\hline Mazama temama & 288 & 20,90 & 3,77 & 41,07 & 31,73 & 13,74 & 24,18 & 13,37 \\
\hline Cuniculus paca & 280 & 20,32 & 8,81 & 6,16 & 54,40 & 65,20 & 0,39 & \\
\hline Panthera onca & 157 & 11,40 & & 3,08 & 3,63 & 27,80 & 8,10 & 3,09 \\
\hline Puma concolor & 147 & 10,67 & & 11,29 & 2,72 & 11,51 & 12,47 & 2,06 \\
\hline Didelphis marsupialis & 126 & 9,15 & 5,03 & 4,11 & 21,76 & 29,72 & 0,13 & \\
\hline Pecari tajacu & 101 & 7,33 & & 13,35 & 31,73 & 9,27 & 3,09 & 1,03 \\
\hline Dasypus novemcinctus & 98 & 7,11 & 74,21 & 13,35 & 4,53 & 4,15 & & 4,12 \\
\hline Nasua narica & 97 & 7,04 & 11,32 & 22,59 & 19,04 & 9,27 & 2,40 & 1,03 \\
\hline Leopardus tigrinus & 81 & 5,88 & & & 0,91 & 4,15 & 2,57 & 6,17 \\
\hline Philander opossum & 74 & 5,37 & 47,80 & 16,43 & 18,13 & & & \\
\hline Conepatus semistriatus & 47 & 3,41 & 3,77 & 3,08 & 9,07 & 6,39 & 1,41 & \\
\hline Eira barbara & 40 & 2,90 & 2,52 & 10,27 & 5,44 & 2,88 & 1,67 & \\
\hline Coendou mexicanus & 39 & 2,83 & & & & 12,46 & & \\
\hline Leopardus wiedii & 35 & 2,54 & 3,77 & 8,21 & 7,25 & 3,84 & & 3,09 \\
\hline Leopardus pardalis & 31 & 2,25 & & & 0,91 & 0,64 & 11,32 & 6,17 \\
\hline Bassariscus sumichrasti & 23 & 1,67 & & & & 0,96 & 2,57 & \\
\hline Dasyprocta punctata & 21 & 1,52 & 13,84 & 1,03 & 5,44 & 0,96 & & \\
\hline Canis latrans & 12 & 0,87 & & 1,03 & & 2,88 & 0,26 & \\
\hline Mustela frenata & 9 & 0,65 & & & 3,63 & 0,32 & 0,51 & \\
\hline $\begin{array}{l}\text { Herpailurus } \\
\text { yagouaroundi }\end{array}$ & 5 & 0,36 & 1,26 & & 0,91 & 0,96 & & \\
\hline Tayassu pecari & 5 & 0,36 & & 2,05 & 1,81 & & 0,13 & \\
\hline Ateles geoffroyi & 1 & 0,07 & & & & 0,32 & & \\
\hline Cebus capucinus & 1 & 0,07 & 1,26 & & & & & \\
\hline Microsciurus alfari & 1 & 0,07 & & & & & & 0,51 \\
\hline
\end{tabular}

*n: número de eventos independientes **IAR: Índice de Abundancia Relativa. BmH-P: Bosque Muy húmedo Premontano. BmHP-TP: Bosque Muy húmedo Premontano Transición a Pluvial. BP-P: Bosque Pluvial Premontano BP-MB: Bosque Pluvial Montano Bajo. BP-M: Bosque Pluvial Montano. BP-S: Bosque Pluvial Subaplino 


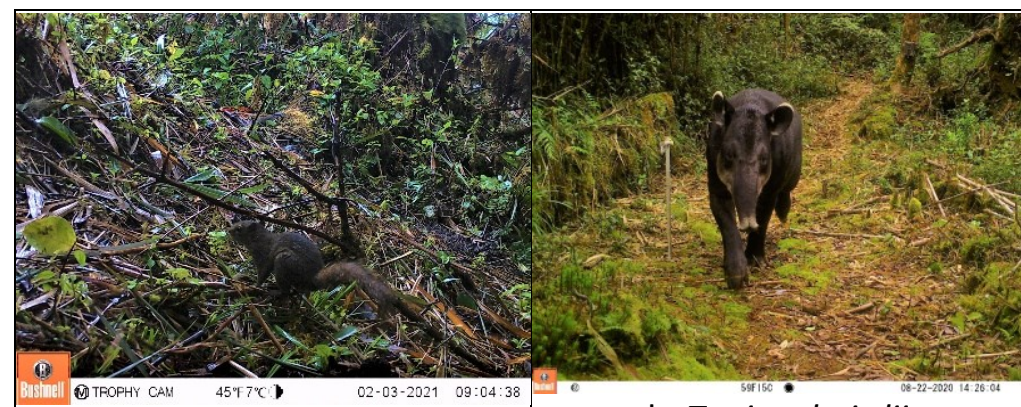

a. Sciurus granatensis

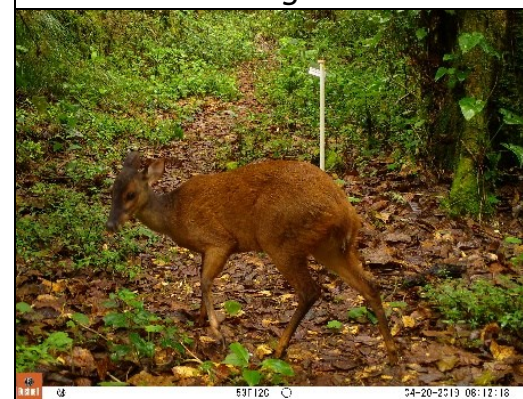

d. Mazama temama

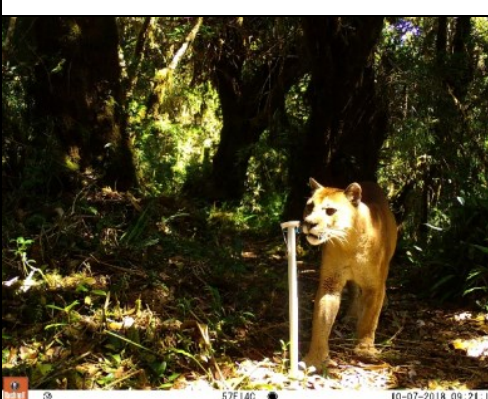

g. Puma concolor b. Tapirus bairdii

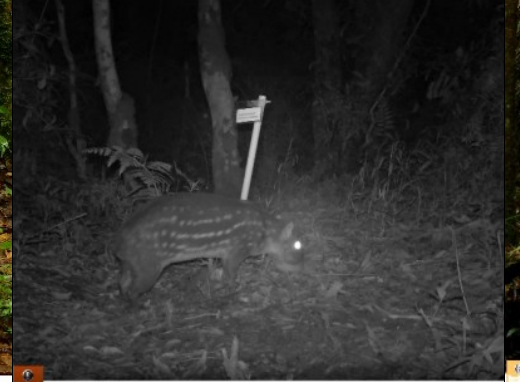

e. Cuniculus paca c. Sylvilagus dicei

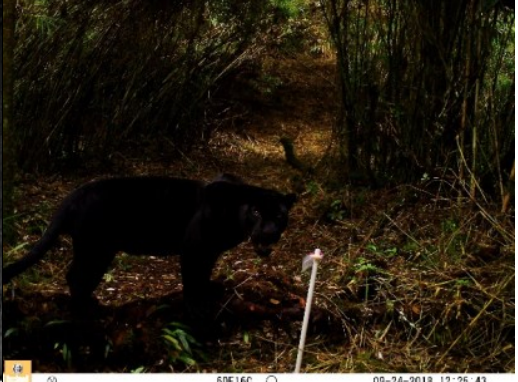

f. Panthera onca (melánico)

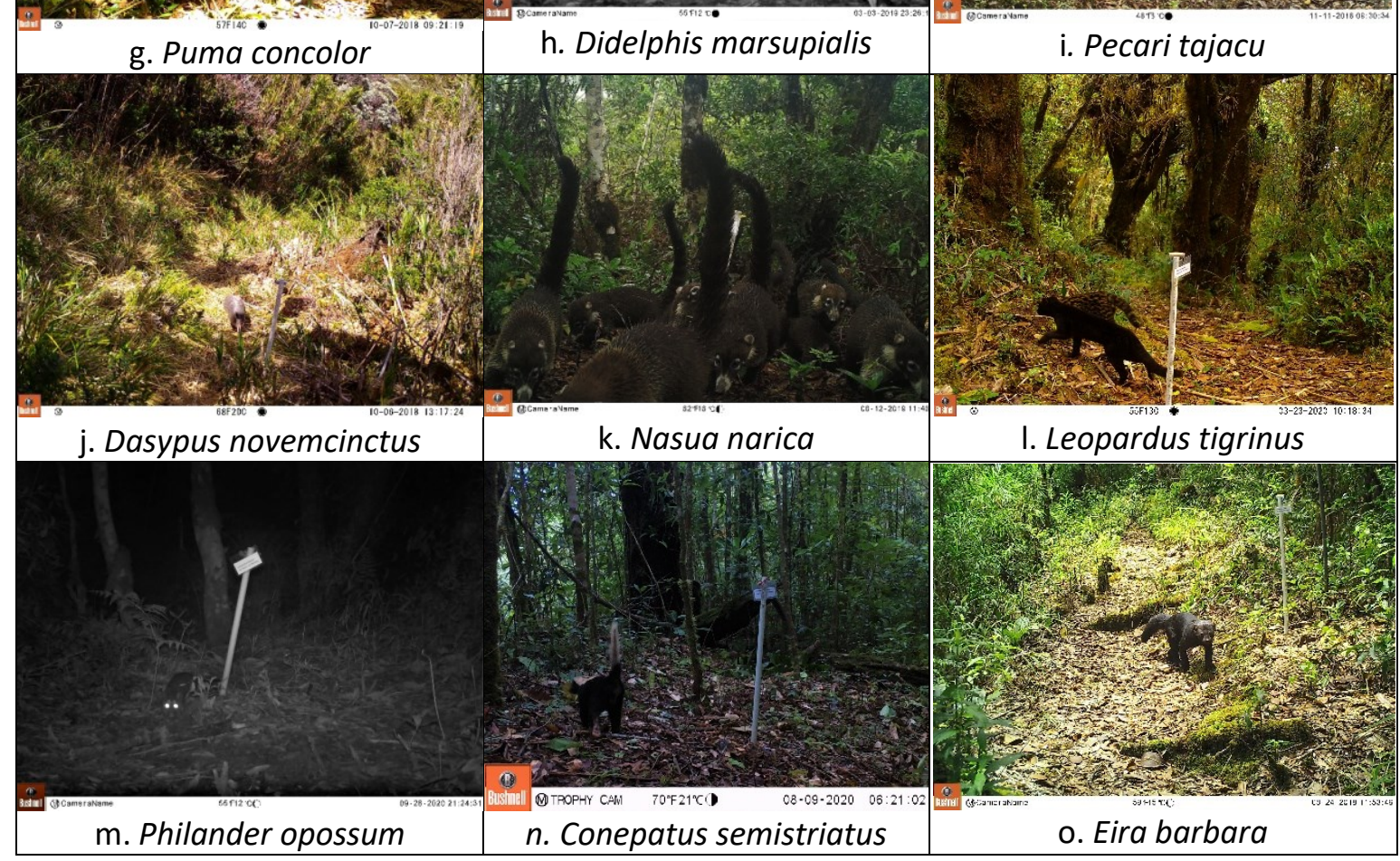

Fig. 3. Especies con la mayor abundancia relativa ordenadas en orden decreciente 
Uso del espacio: Detectamos la mayor riqueza (23 especies) en el BP-MB y la menor riqueza (12 especies) en el BP-S. Las especies con mayor distribución por zona de vida y piso altitudinal fueron S. granatensis, T. bairdii, $M$. temama y $N$. narica. Las especies con la distribución más restringida fueron $C$. imitator, $C$. mexicanus, A. geoffroyi y $M$. alfari (TABLA 1). Detectamos individuos manchados y melánicos de $P$. onca y $L$. tigrinus. Los jaguares melánicos los detectamos en siete zonas de vida ubicadas en el CBQTC (BmHP-TP, BP-MB) y en el PILA (BP-M, BP-S). La coloración melánica de $L$. tigrinus la detectamos en tres estaciones ubicadas en el PILA (BP-MB, BP$\mathrm{M}, \mathrm{BP}-\mathrm{S}$ ). EI PILA y el CBQTC comparten 22 especies en común; dos especies se registraron solo en PILA ( $C$. mexicanus y $M$. alfari) y tres especies se registraron únicamente en CBQTC ( $A$. geoffroyi, $C$. imitator, $P$. opossum). Registramos cinco eventos independientes de Tayassu pecari, en las zonas de vida BmHP-TP, BP-P y BP-M.

Patrones de actividad: Detectamos seis especies diurnas (M. frenata, E. barbara, $A$. geoffroyi, $C$. imitator, S. granatensis, M. alfari), siete nocturnas y crepusculares ( $D$. marsupialis, $C$. paca, B. sumichrasti, C. mexicanus, C. semistriatus, P. oposum, T. pecari) y 14 catemerales (P. tajacu, T. bairdi, S. dicei, N. narica, M. temama, L. pardalis, L. tigrinus, L. wiedii, P. concolor, P. onca, $H$. yagouaroundi, $D$. novemcinctus, $D$. punctata, $C$. latrans). De las especies que mostraron actividad nocturna, seis (B. sumichrasti, C. mexicanus, C. semistriatus, S. dicei, M. temama y C. latrans) mostraron filia lunar, ocho (C. paca, P. tajacu, L. pardalis, L. tigrinus, H. yagouaroundi, D. novemcinctus, $N$. narica, $D$. punctata) mostraron fobia lunar y seis especies (D. marsupialis, $P$. oposum, T. bairdi, L. wiedii, $P$. concolor y $P$. onca) no mostraron afinidad al brillo lunar. Analizamos el IAR, el $\boldsymbol{\Delta}$ y las pruebas estadísticas ( $U^{2}$ de Watson, $W_{r}$ y Fisher) de las especies con $\mathbf{n}>\mathbf{2 0}$ con respecto a la presencia de personas y mamíferos domésticos (Apéndice digital, TABLA 4). La mayor similitud en la actividad circadiana de las especies silvestres ocurrió con las vacas y la mayor diferencia con los caballos. Dos especies (E. barbara y D. punctata) mostraron la mayor similitud de actividad circadiana con C. familiaris (Apéndice digital, Fig. 4 a la 23).

Los resultados sugieren que el patrón de actividad de cada especie no mostró diferencia significativa entre la estación seca y la lluviosa (Apéndice digital, TABLA 5). La cantidad de eventos independientes fue significativamente diferente para 12 especies ( $C$. mexicanus, $C$. semistriatus, $D$. marsupialis, P. opossum, D. novemcinctus, P. concolor, L. pardalis, L. tigrinus, P. onca, P. tajacu, N. narica, $D$. punctata) y no fue significativamente diferente para 7 especies (B. sumichrasti, S. dicei, $L$. wiedii, M. temama, T. bairdii, S. granatensis, E. barbara). Durante la estación seca (2019-2021) ingresaron 301 turistas al Sendero Tres Colinas-Cerro Kamuk y 214 finqueros y turistas al CBQTC. Debido a la emergencia sanitaria ocasionada por el COVID-19, el PILA permaneció cerrado a la visitación desde el 23 de marzo del 2020; los visitantes ingresaron a partir del 1 de diciembre del 2020 con un aforo del 50\% (Apéndice digital, TABLA 6).

\section{DISCUSIÓN}

Riqueza y estado de conservación: Mediante el fototrampeo detectamos 27 especies silvestres; de acuerdo con Silveira et al. (2003), esto representa cerca del $70 \%$ de la riqueza de nuestra población y sitio de estudio. Podríamos detectar otras especies si prolongamos el tiempo de muestreo y aumentamos la cantidad de cámaras trampa (Karanth et al., 2004a; Ahumada et al., 2011) instaladas en el borde de pasos antrópicos (Piedra \& Maffei, 2000; Karanth et al., 2004b; Blake et al., 2017; Tan et al., 2018), ya que por diversos factores (Karanth et al., 2004a; Arroita \& Monfort, 2015) la detección de mamíferos silvestres varía temporal y espacialmente (O’Connell et al., 2011). 
Todas las especies detectadas están en las listas de la UICN, CITES o MINAE, lo cual refleja la importancia de conservación del sitio. Herpailurus yagouaroundi, M. frenata, C. latrans, L. pardalis y $L$. wiedii muestran un bajo número de detecciones debido a que, por su naturaleza, son raras y elusivas (Tobler et al., 2008). La presencia de carnívoros como C. latrans y seis especies de felinos silvestres, indican un buen estado de conservación de los ecosistemas del PILA y del CBQTC, pues son sensibles a la modificación de la cobertura vegetal (Dirzo et al., 2014) y requieren espacios amplios (Holden \& Neang, 2009; Cueva et al., 2010). Dichos ecosistemas son aptos para la conservación de especies a largo plazo (Kappelle, 2004; Avalos, 2007), algunas de las cuales ( $A$. geoffroyi, H. yagouaroundi, L. pardalis, L. tigrinus, L. wiedii, P. concolor, P. onca, T. bairdii, C. paca y T. pecari) tienen poblaciones amenazadas o están en peligro de extinción (Rodríguez et al., 2002).

Abundancia relativa: Tapirus bairdii obtuvo el más alto valor de conservación y fue la segunda especie más abundante $(I A R=53,42)$, similar a lo detectado por Brenes (2018). Sy/vilagus dicei fue la tercera especie más abundante (IAR=25,91); al ser una especie endémica de Talamanca (Wainwright, 2007), depende por completo de un área específica y es mucho más vulnerable a los cambios en la composición vegetal (Kappelle, 2008). Los constantes registros $(I A R=3,09)$ de $P$. onca melánico en el BP-S a $3142 \mathrm{msnm}$ es novedoso, pues dicha especie es rara en elevaciones superiores a 2000 msnm (González et al., 2008). Los múltiples registros melánicos de $P$. onca y L. tigrinus siguieron el mismo patrón de actividad descrito por Mooring et al. (2020).

Uso del espacio: Hay una considerable similitud de especies en el PILA que también están en el CBQTC, sin embargo, la diversidad de especies disminuye conforme aumenta la elevación de los pisos altitudinales (Ramos et al., 2007) desde el CBQTC hasta el PILA. Obtuvimos la mayor riqueza en los pisos altitudinales Premontano y Montano Bajo (CBQTC), lo cual indica un buen estado de conservación con diversos grupos funcionales y sin dominancia de pocas especies (Ahumada et al., 2011). Tanto por zona de vida como por piso altitudinal, las especies con la distribución más amplia fueron $T$. bairdii, $M$. temama, $N$. narica y $S$. granatensis. Las especies con la distribución más restringida fueron $B$. sumichrasti, A. geoffroyi, $C$. imitator, $C$. mexicanus y $M$. alfari. A excepción de S. granatensis, se evidenció que el tamaño corporal grande está relacionado con un amplio rango de hogar y densidad (Bakker et al., 2000; Trejo et al., 2010; Ahumada et al., 2011) y se debe considerar su interacción constante con el ecosistema (Maciel et al., 2015). Dos de las elevaciones donde registramos T. pecari coinciden con la distribución descrita por Carrillo et al. (2002) y Wainwright (2007). Sin embargo, detectamos esta especie a $2768 \mathrm{msnm}$ lo cual es poco usual.

Patrones de actividad: El tamaño corporal de P. tajacu, T. bairdi, S. dicei, N. narica, $M$. temama, L. pardalis, L. tigrinus, L. wiedii, P. concolor, P. onca, H. yagouaroundi, D. novemcinctus, $D$. punctata, $C$. latrans les permite alimentarse durante el día y la noche a diferencia de las especies que se sólo se alimentan durante el día (M. frenata, E. barbara, A. geoffroyi, C. imitator, M. alfari) o la noche (D. marsupialis, C. paca, B. sumichrasti, C. mexicanus, C. semistriatus, $P$. oposum)(Emmons et al., 1983). Detectamos varias presas potenciales (Botts et al., 2020a) entre ellas seis especies unguladas, que indican una población saludable de depredadores grandes pues éstos seleccionan presas igualmente grandes (Tan et al., 2018). Se detectaron especies diurnas, nocturnascrepusculares y catemerales, pero ninguna fue estrictamente crepuscular (Van Schaik y Griffiths, 1996; Kirk, 2006; Bennie et al., 2014). Estos patrones de actividad obedecen a la morfología y fisiología del sistema visual (Kirk, 2004), a aspectos ecológicos (Di Bitetti et al., 2009), al rol de presas y depredadores (Emmons et al., 1983; Prugh y Golden, 2014; Suselbeek et al., 2014), a la alimentación (Downes, 2001) y al estrés térmico (Owen, 1998). 
Algunas especies no mostraron afinidad al brillo lunar (Norris et al., 2010; Penteriani et al., 2013), otras especies mostraron filia (Lizcano \& Cavelier, 2000) o fobia (Harmsen et al., 2011); sin embargo, estos patrones de actividad varían incluso entre individuos de la misma especie (Coelho et al., 2008; Oliveira et al., 2010; Harmsen et al., 2011; Michalski \& Norris, 2011). En noches con luna llena C. paca, P. tajacu, D. novemcinctus, N. narica y D. punctata no forrajearon para evitar depredadores (Kotler et al., 2010), mientras que L. pardalis, L. tigrinus y H. yagouaroundi evitaron competir con otros predadores mayores (Botts et al., 2020b). Durante la estación seca, L. pardalis mostró mayor actividad en la tarde, quizá aprovechando las horas del día sin lluvia para buscar alimento, aunque Moreno et al. (2012) determinaron que esta especie fue mayormente nocturna $(63,2 \%)$. Pecari tajacu y $M$. temama disminuyeron su actividad en el medio día, posiblemente para evitar el estrés térmico (Owen, 1998; Oliveira et al., 2010).

La regeneración natural, los parches boscosos y el abandono de tierras ha favorecido el turismo ecológico y la conservación de los mamíferos (Rodríguez et al., 2002). Éstos desempeñan un importante papel en la estructuración y regeneración de los ecosistemas (Escribano et al., 2015). Recomendamos continuar restaurando la conectividad biológica a través de estrategias como el CBQTC, el cual ha disminuido la deforestación provocada por la agricultura, ganadería e incendios forestales (Martínez, 2013). Concluimos que la semejanza de 22 especies entre el CBQTC y el PILA obedece a la continuidad y gran área boscosa del ASP, lo cual favorece la permanencia y el tránsito de mamíferos silvestres (Sridhari et al., 2008) en el CBQTC y de ahí a otras áreas adyacentes, donde son vulnerables a la cacería y entran en conflicto con los seres humanos (Arias et al., 2008; Céspedes et al., 2008; Cartín \& Carrillo, 2017). Recomendamos enfocar acciones de protección a especies como T. pecari, la cual se está movilizando a tierras más altas probablemente por la presión que enfrenta fuera del ASP (Sáenz \& Carrillo, 2002).

Para todas las especies silvestres, la mayor similitud en el $\boldsymbol{\Delta}$ de su actividad circadiana se observó con B. taurus; la mayor diferencia ocurrió con $E$. caballus, sin embargo, debido a su pequeña muestra $(n=12)$ este resultado debe interpretarse de manera conservadora. De acuerdo con el $\Delta$, la mayor probabilidad de encuentros físicos ocurriría entre los mamíferos silvestres y $B$. taurus. El mayor valor de superposición $(0,90)$ ocurrió entre $N$. narica y $\mathrm{H}$. sapiens, por lo cual es posible que los visitantes puedan observarlos. Existe un gran riesgo de zoonosis entre los mamíferos domésticos y humanos hacia los mamíferos silvestres y viceversa (Acha \& Szyfres, 2001; Monsalve et al., 2009), por lo cual recomendamos tomar las medidas pertinentes para impedir el paso de mamíferos domésticos hacia el PILA.

Aunque hubo variación en la cantidad de eventos independientes entre las estaciones seca y lluviosa (Santos et al., 2010), el patrón de actividad no fue significativamente diferente. Las estadísticas indican que la riqueza y abundancia de mamíferos silvestres no parece ser afectada por la presencia de humanos y mamíferos domésticos. Concluimos que las regulaciones a la visitación turística (Brenes et al., 2004; SINAC, 2019) han sido una herramienta técnica adecuada para conservar las especies silvestres y recomendamos que éstas se mantengan. Esta investigación es una línea base previo al desarrollo turístico del Sendero Tres Colinas-Cerro Kamuk. Recomendamos continuar con el fototrampeo a largo plazo, para medir el impacto del turismo en los mamíferos silvestres antes, durante y después de la construcción de infraestructura turística. 


\section{AGRADECIMIENTOS}

Agradecemos a la Universidad de Point Loma Nazarene, Centro de Educación e Investigación Quetzal, Asociación de Turismo de Tres Colinas, Sistema Nacional de Áreas de Conservación, Freddy Acuña, Yendry Rojas, Rony Rojas, Ania Jiménez, Jim Sanderson, Esteban Brenes, Stephanny Arroyo, Claudine Sierra, Emma Mooring, Sergio Quesada, Zaidett Barrientos, Gravin Villegas, Ronald Chan, Andrés y Arturo Mora, Óscar Solano, Iván Leitón, Junior Porras y Ronnie Chong. Este trabajo es parte de los requisitos de graduación del programa de Maestría Académica en Manejo de Recursos Naturales de la Universidad Estatal a Distancia de Costa Rica.

\section{ÉTICA, CONFLICTO DE INTERESES Y DECLARACIÓN DE FINANCIAMIENTO}

Declaramos haber cumplido con todos los requisitos éticos y legales pertinentes, tanto durante el estudio como en la preparación de este documento; que no hay conflictos de interés de ningún tipo, y que todas las fuentes financieras se detallan plena y claramente en la sección de agradecimientos. Asimismo, estamos de acuerdo con la versión editada final de esta publicación. El respectivo documento legal firmado se encuentra en los archivos de la revista.

La declaración de contribución de cada autor es la siguiente: R.G.T.: Diseño del estudio, recolección, recopilación de datos, preparación del manuscrito. A.E.: Análisis de datos y elaboración de fotogramas. M.M. Revisión, corrección y aprobación final del manuscrito. Todos los coautores: revisión de fotografías e identificación de especies.

\section{REFERENCIAS}

Acha, P., \& Szyfres, B. (2001). Zoonosis y enfermedades transmisibles comunes al hombre y a los animales. Organización Panamericana de la Salud. https://iris.paho.org/bitstream/handle/10665.2/710/9275319928.pdf

Agafonkin, V., \& Thieurmel, B. (2018). Suncalc: Compute sun position, sunlight phases, moon position and lunar phase. (version 0.4) [software]. R package. https://cran.r-project.org/web/packages/suncalc/suncalc.pdf

Ahumada, J., Hurtado, J., \& Lizcano, D. (2013). Monitoring the status and trends of tropical forest terrestrial vertebrate communities from camera trap data: a tool for conservation. PLoS ONE, 8(9), 1-10. https://doi:10.1371/journal.pone.0073707

Ahumada, J., Silva, C., Gajapersad, K.; Hallam, C.; Hurtado, J., Martin, E.; McWilliam, A.; Mugerwa, B.; Brien, T., Rovero, F.; Sheil, D.; Spironello, W.; Winarni, N., \& Andelman, S. (2011). Community structure and diversity of tropical forest mammals: data from a global camera trap network. The Royal Society, 366, 2703-2711. https://doi:10.1098/rstb.2011.0115

Altrichter, M., \& Carbonell, F. (2013). Efectos de la cacería en la Reserva Indígena Talamanca Bribrí-Cabécar e importancia del Parque Internacional de La Amistad, Costa Rica. Revista Latinoamericana de Conservación, 3(2), 38-47.

Anile, S., \& Devillard, S. (2016). Study design and body mass influence RAls from camera trap studies: Evidence from theFelidae. Animal Conservation, 19, 35-45. https://doi.org/10. 1111/acv.12214

Arias, E., Chacón, O., Herrera, B., Induni, G., Acevedo, H., Coto, M. \& Barborak, J. (2008). Las redes de conectividad como base para la planificación de la conservación de la biodiversidad: propuesta para Costa Rica. Recursos Naturales y Ambiente, 54, 37-43.

Arita, H., \& Figueroa, F. (1999). Geographic patterns of body-mass diversity in Mexican Mammals. Oikos, 85(2), 310-319. https://doi.org/10.2307/3546497 
Arroita, G., \& Monfort, J. (2015). El efecto de la detección imperfecta en el modelado de distribución de especies. University of Melbourne. Decision Point. E01, 20-21.

Avalos, G. (2007). Reseña de páramos de Costa Rica de M. Kappelle y S. P. Horn. Revista de Biología Tropical, 55, (2), 743744. https://doi.org/10.15517/rbt.v55i2.6050

Azuara, S. (2005). Estimación de abundancia de mamíferos terrestres en un área de la Selva Lacandona, Chiapas [Tesis de Licenciatura, Universidad Autónoma de México].

Bakker, V., \& Kelt, D. (2000). Scale-dependent patterns in body size distributions of neotropical mammals. Ecology, 81(12), 3530-3547. https://doi.org/10.1890/0012-9658(2000)081[3530:SDPIBS]2.0.CO;2

Barea, J., Virgós, E., Ballesteros, E., Moleón, M., \& Chirosa, M. (2007). Surveying carnivores at large spatial scales: A comparison of four broad-applied methods. Biodiversity and Conservation, 16, 1213-1230. https://doi.org/10.1007/s10531-006-9114-x

Bennie, J., Duffy, J., Inger, R., \& Gaston, K. (2014). Biogeography of time partitioning in mammals. Biological Sciences, 111, 13727-13732. https://doi.org/10.1073/pnas.1216063110

Berger-Tal, O.; Polak, P.; Oron, A.; Lubin, Y.; Kotler, B., \& Saltz, D. (2011). Integrating animal behavior and conservation biology: a conceptual framework. Behavioral Ecology, 22(2), 236-239. https://doi:10.1093/beheco/arq224

Blake, J., Mosquera, D., Loiselle, B., Romo, D., \& Swin, K. (2017). Effects of human traffic on use of trails by mammals in lowland forest of eastern Ecuador. Neotropical Biodiversity, 3(1), 57-64. https://doi.org/10.1080/23766808.2017.1292756

Botts, R., Eppert, A., Wiegman, T., Rodriguez, A., Blankenship, S., Asselin, E., Garley, W, Wagner, A., Ullrich, S., Allen, G., \& Mooring, M. (2020a). Circadian activity patterns of mammalian predators and prey in Costa Rica. Journal of Mammalogy, 101(5), 1313-1331. https://doi.org/10.1093/imammal/gyaa103

Botts, R., Eppert, A., Wiegman, T., Blankenship, S., Rodriguez, A., Wagner, A., Ullrich, S., Allen, G., Garley, W., Asselin, E., \& Mooring, M. (2020b). Does moonlight increase predation risk for elusive mammals in Costa Rica? Tropical Conservation Science, 13, 1-21. https://doi.org/: 10.1177/1940082920952405

Braczkowski, A., \& Watson, L. (2013). Observations of leopard and caracal responses to novel scents in South Africa. CATnews, 58, 13-15.

Braczkowski, A., Balme, G., Dickman, A., Fattebert, J., Johnson, P., Dickerson, T., \& Hunter, L. (2016). Scent lure effect on camera-trap based leopard density estimates. PLoS ONE, 11(4), 114 https://doi.org/10.1371/journal.pone.0151033

Brenes, E. (2018). Patrones de actividad, selección de hábitat y atropellos de danta (Tapirus bairdii) en bosque atravesado por una carretera en la Cordillera de Talamanca, Costa Rica. [Tesis de Maestría, Universidad Nacional de Costa Rica].

Brenes, O., Castro, K., Jiménez, V., \& Mora, A. (2004). Determinación de la capacidad de carga turística del Parque Internacional de La Amistad. Centro Científico Tropical-The Nature Conservancy. https://bit.ly/3w7vyZx

Calvo, A. (2009). Determinación de índices de fragmentación y modelamiento de la conectividad en los corredores biológicos de Costa Rica [Tesis de grado, Instituto Tecnológico de Costa Rica].

Carbonell, F., \& Torrealba, I. (2007). Conservación en Ecotonos Interculturales y Transfronterizos: La Danta (Tapirus bairdii) en el Parque Internacional La Amistad, Costa Rica-Panamá. Tapir Conservation, 16(1), 24-30.

Carrillo, E., Wong, G., \& Sáenz J. (2002). Mamíferos de Costa Rica. Editorial Instituto Nacional de Biodiversidad. 
Carter, N., Shrestha, B., Karki, J., Pradhan, N., \& Liu, J. (2012). Coexistence between wildlife and humans at fine spatial scales. Proceedings of the National Academy of Sciences, 109(38), 15360-15365 https://doi.org/10.1073/pnas.1210490109

Cartín, M., \& Carrillo, E. (2017). Estado poblacional de mamíferos terrestres en dos Áreas Protegidas de la región central occidental de Costa Rica. Revista de Biología Tropical, 65(2), 493-503. https://doi.org/10.15517/rbt.v65i2.24418

Carver, B., Kennedy, M., Houston, A., \& Franklin, S. (2011). Assessment of temporal partitioning in foraging patterns of syntopic Virginia opossums and raccoons. Journal of Mammalogy, 92(1), 134-139. https://doi.org/10.1644/10MAMM-A-066.1

Ceballos, G., \& Navarro, D. (1991). Diversity and conservation of Mexican mammals. Mares \& Schmidly, Latin American Mammalogy (1 ed., pp. 167-198). University of Oklahoma Press. https://bit.ly/3pUmm9F

Céspedes, M., Finegan, B., Herrera, B., Delgado, L., Velásquez, S., \& Campos, J. (2008). Diseño de una red ecológica de conservación entre la Reserva de Biosfera La Amistad y las Áreas Protegidas del Área de Conservación Osa, C.R. Recursos Naturales y Ambiente, 54, 44-50.

Chaverri, G., Garin, I., Alberdi, A., Jiménez, L., Castillo, C., \& Aihartza, J. (2016). Unveiling the Hidden Bat Diversity of a Neotropical Montane Forest. PloS ONE, 11(10) 1-19. https://doi.org:10.1371/journal.pone.0162712

Coelho, I., Oliveira, L., \& Oliveira, M. (2008). Does moonlight affect the use of natural licks by lowland tapir (Tapirus terrestris Linnnaeus, 1758) in the Northeastern Brazilian Pantanal? Tapir Conservation, 17/2 (24), 10-14.

Cortés, M., \& Briones, M. (2014). Diversidad, abundancia relativa y patrones de actividad de mamíferos medianos y grandes en una selva seca del Istmo de Tehuantepec, Oaxaca, MéxicoRevista de Biología Tropical, 62(4), 14331448. https://doi.org/10.15517/rbt.v62i4.13285

Cuellar, E., Maffei, L., Arispe, R., \& Noss, A. (2006). Geoffroy's cats at the northern limit of their range: activity patterns and density estimates from camera trapping in Bolivian dry forests. Studies of Neotropical Fauna Environmental, 41(3), 169-177. https://doi.org: 10.1080/01650520600840001

Cueva, X., Morales, N., Brown, M., \& Peck, M. (2010). Macro y mesomamíferos de la Reserva Comunitaria Santa Lucía, Pichincha, Ecuador. Serie Zoológica, 6: 98-110.

Di Bitetti, M., Di Blanco, Y., Pereira, J., Paviolo, A., \& Pérez, I. (2009). Time partitioning favors the coexistence of sympatric crab-eating foxes (Cerdocyon thous) and pampas foxes (Lycalopex gymnocercus). Journal of Mammalogy, 90, 479490. https://doi.org/10.1644/08-MAMM-A-113.1

Dirzo, R., Broadbent, E., Almeyda, A., Morales, L., Almeyda, S.; Zambrano, A., \& Quispe, C. (2014). Especies indicadoras del estado de conservación de Osa y Golfito. Stanford Woods Institute for the Environment Stanford University. https://inogo.stanford.edu/sites/default/files/Especies\%20Indicadoras\%20Final\%20INOGO\%202014.pdf

Downes, S. (2001). Trading heat and food for safety: costs of predator avoidance in a lizard. Ecology, 82, $2870-2881$. https://doi.org/10.1890/0012-9658(2001)082[2870:THAFFS]2.0.CO;2

Emmons. L., Gautier, A., \& Dubost, G. (1983). Community structure of the frugivorous-folivorous forest mammals of Gabon. Journal of Zoology, 199, 209-222. https://doi.org/10.1111/i.1469-7998.1983.tb02091.x

Escribano, G., Pías, B., Escudero, A., \& Virgós, E. (2015). Importancia ecológica de los mamíferos frugívoros en la dinámica de regeneración de campos abandonados en ambientes mediterráneos. Ecosistemas, 24(3), 35-42. https://doi.org/10.7818/ECOS.2015.24-3.06

Fisher, N. (1993). Statistical Analysis of Circular Data. Cambridge University Press. https://doi.org/10.1017/CBO9780511564345 
Florian, E., Harvey, C., Finegan, B., Benjamín, T., \& Soto, G. (2010). Efecto de la complejidad estructural y el contexto paisajístico en la avifauna de sistemas agroforestales cafetaleros dentro del Corredor Biológico Volcánica CentralTalamanca. Mesoamericana, 14(3), 66-72.

García, R. (2012). Desempeño del perfume Obsession en el estudio de jaguares (Felidae: Carnivora) con cámaras automáticas en la Reserva de la Biosfera Maya. [Tesis de Maestría, Universidad de San Carlos de Guatemala].

Gerber, B., Karpanty, S., \& Kelly, M. (2012). Evaluating the potential biases in carnivore capture-recapture studies associated with the use of lure and varying density estimation techniques using photographic-sampling data of the Malagasy civet. Population Ecology, 54(1), 43-54. https://doi.org/10.1007/s10144-011-0276-3

Gibeau, M., \& McTavish, C. (2009). Not-so-candid cameras: How to prevent camera traps from skewing animal behavior. The Wildlife Society Professional, Fall. 35-37.

Gómez, H., Wallace, R., Ayala, G., \& Tejada, R. (2005). Dry season activity periods of some Amazonian mammals. Studies on Neotropical Fauna and Environment, 40, 91-95. https://doi.org/10.1080/01650520500129638

González, J., Finegan, B., Schipper, J., \& Casanoves, F. (2008). Densidad absoluta y conservación del jaguar y sus presas en la Región Talamanca Pacífico, Costa Rica. (Serie Apoyando los esfuerzos en el manejo y protección de la biodiversidad tropical $N^{\circ} 7$ ). The Nature Conservancy. https://bit.ly/2ZJkx50

Griffiths, M., \& Van Schaik, C. (1993). Camera-trapping: a new tool for the study of elusive rain forest animals. Tropical Biodiversity, 1, 131-135.

Harmsen, B., Foster, R., Silver, S., Ostro, L., \& Doncaster, C. (2009). Differential use of trails by forest mammals and the implications for camera-trap studies: a case study from Belize. Biotropica, 42(1), 126-133. https://doi.org/10.1111/i.1744-7429.2009.00544.x

Harmsen, B., Foster, R., Silver, S., Ostro, L., \& Doncaster, C. (2011). and puma activity patterns in relation to their main prey. Mammalian Biology, 76, 320-324. https://doi.org/10.1016/j.mambio.2010.08.007

Hernández, E., Reyna, R., Castillo, G., Sanvicente, M., \& Moreira, J. (2015). Fototrampeo de mamíferos terrestres de talla mediana y grande asociados a petenes del noroeste de la península de Yucatán, México. Therya, 6 (3), 559-574. https://doi.org/10.12933/therya-15-290

Holden, J., \& Neang, T. (2009). Small carnivore records from the Cardamom Mountains, southwestern Cambodia. Small Carnivore Conservation, 40, 16-21.

Holdridge, L. (1987). Ecología basada en zonas de vida. Instituto Interamericano de Ciencias Agrícolas. http://www.cct.or.cr/contenido/wp-content/uploads/2017/11/Ecologia-Basada-en-Zonas-de-Vida-Libro-IV.pdf

Hurtado, J., \& Soto, C. (2017). Manual para el monitoreo participativo de vertebrados terrestres a través de cámaras trampa en Costa Rica. Proyecto MAPCOBIO-SINAC-JICA. https://bit.ly/3jTh9el

Hurtado, J., Osawa, M., Artavia, A., Gutiérrez, G., Vásquez, P., Jiménez, M., Mory, S., Bonilla, S., Rodríguez, M., Corrales, D., Orrego, C., Vindas, J., Quirós, J., \& Arce, A. (2018). Uso de cámaras trampa en Costa Rica y sus aplicaciones para el manejo y conservación de la vida silvestre. Proyecto MAPCOBIO-SINAC-JICA. https://bit.ly/3jVrOFJ

Jaime, N.; Figueroa, E.; Villaseñor, J.; Jacobo, E., \& Puebla, F. (2016). Distribución altitudinal de la riqueza y composición de "ensamblajes" de aves en una zona montañosa al sur de Nayarit, México. Revista Biología Tropical, 64 (4), 15371551. https://doi.org/10.15517/rbt.v64i4.20255

Janecka, J., Munkhtsog, B., Jackson, R., Naranbaatar, G., Mallon, D., \& Murphy, W. (2011). Comparison of noninvasive genetic and camera-trapping techniques for surveying snow leopards. Journal of Mammalogy, 92(4), 771-783. https://doi.org/10.1644/10-MAMM-A-036.1

Jenks, K., Chanteap, P., Damrongchainarong, K., Cutter, P., Cutter, P., Redford, T., Lynam, A., Howard, J., \& Leimgruber, P. (2011). Using relative abundance indices from camera-trapping to test wildlife conservation hypotheses - an 
example from Khao Yai National Park, Thailand. Tropical Conservation Science, 4, 113-131. https://doi.org/10.1177/194008291100400203

Jinn, J. (2019). Orientation and sampling strategies in mammalian olfactory navigation [Tesis de doctorado, University of California, Berkeley, California].

Kamler, J., Johnson, A., Vongkhamheng, C., \& Bousa, A. (2012). The diet, prey selection, and activity of dholes (Cuon alpinus) in northern Laos. Journal of Mammalogy, 93(3), 627-633. https://doi.org/10.1644/11-MAMM-A-241.1

Kappelle, M., \& Brown, A. (2001). Bosques nublados del Neotrópico. Editorial Instituto Nacional de Biodiversidad.

Kappelle, M., \& Horn, S. (2005). Páramos de Costa Rica. Editorial Instituto Nacional de Biodiversidad.

Kappelle, M. (1996). Los bosques de roble (Quercus) de la Codillera de Talamanca, Costa Rica. Biodiversidad, ecología y conservación. Editorial Instituto Nacional de Biodiversidad.

Kappelle, M. (2004). Tropical montane forests. Encyclopedia of Forest Sciences, 4, 1782-1793. https://doi.org/10.1016/B012-145160-7/00175-7

Kappelle, M. (2008). Diccionario de la biodiversidad. Editorial Instituto Nacional de Biodiversidad.

Karanth, K., Chundawat, R., Nichols, J., \& Kumar, S. (2004b). Estimation of tiger densities in the tropical dry forests of Panna, Central India, using photographic capture recapture sampling. Animal Conservation,7, 285-290. https://doi.org/10.1017/S1367943004001477

Karanth, K., Nichols, J., Kumar, N., Link, W., \& Hines, J. (2004a). Tigers and their prey: predicting carnivore densities from prey abundance. Proceedings of the National Academy of Sciences, 101(14), 4855-4858. https://doi.org/10.1073/pnas.0306210101

Karanth, K., Nichols, J., Kumar, S., \& Hines, J. (2006). Assessing tiger population dynamics using photographic capturerecapture sampling. Ecology, 87(11), 2925-2937. https://doi.org/10.1890/00129658(2006)87[2925:ATPDUP]2.0.CO;2

Kirk, E. (2004). Comparative morphology of the eyes of primates. The Anatomical Record. 281(A): 1095-1103. https://doi.org/10.1002/ar.a.20115

Kirk, E. (2006). Eye morphology in cathemeral lemurids and other mammals. Folia Primatologica, 77, 27-49. https://doi.org/10.1159/000089694

Kitchen, A., Gese, E., \& Schauster, E. (2000). Changes in coyote activity patterns to reduced exposure to human persecution. Canadian Journal of Zoology, 78, 853-857. https://doi.org/10.1139/cjz-78-5-853

Kotler, B. P., Brown, J., Mukherjee, S., Berger-Tal, O., \& Bouskila, A. (2010). Moonlight avoidance in gerbils reveals a sophisticated interplay among time allocation, vigilance and state-dependent foraging. The Royal Society, 277(1687), 1469-1474. https://doi.org/10.1098/rspb.2009.2036

Kozlov, D. (2018). ReNamer (version 7.3) [software]. Den4b. https://www.den4b.com/

Linkie, M., \& Ridout, M. (2011). Assessing tiger-prey interactions in Sumatran rainforests. Journal of Zoology, 284, 224229. https://doi.org/10.1111/j.1469-7998

Lira, I., \& Briones, M. (2011). Impacto de la ganadería extensiva y cacería de subsistencia sobre la abundancia relativa de mamíferos en la Selva Zoque, Oaxaca, México. Therya, 2, 217-244. https://doi.org/10.12933/therya-11-49.

Lira, I., \& Briones, M. (2012). Abundancia relativa y patrones de actividad de los mamíferos de los Chimalapas, Oaxaca, México. Acta Zoológica Mexicana, 28(3), 566-585. https://doi.org/10.21829/azm.2012.283859 
Lira, I., Briones, M., \& Sánchez, G. (2014). Abundancia relativa, estructura poblacional, preferencia de hábitat y patrones de actividad del tapir centroamericano Tapirus bairdii (Perissodactyla: Tapiridae), en la Selva de Los Chimalapas, Oaxaca, México. Revista de Biología Tropical, 62(4), 1407-1419. https://doi.org/10.15517/rbt.v62i4.12584

Lizcano, D., \& Cavelier, J. (2000). Daily and seasonal activity of the mountain tapir (Tapirus pinchaque) in the Central Andes of Colombia. Journal of Zoology, 252, 429-435. https://doi.org/10.1111/j.1469-7998.2000.tb01225.x

Lynam, A., Jenks, K., Tantipisanuh, N., Chutipong, W., Ngoprasert, D., \& Steinmetz, R., Leimgruber, P. (2013). Terrestrial activity patterns of wild cats from camera-trapping. The Raffles Bulletin of Zoology, 61(1), 407-415.

Maciel, C., Manríquez, N., Octavio, P., \& Sánchez, G. (2015). El área de distribución de las especies: revisión del concepto. Acta Universitaria, 25(2), 3-19. https://doi.org/10.15174/au.2015.690

Maffei, L., Cuellar, E., \& Noss, J. (2002). Uso de trampas cámara para la evaluación de mamíferos en el ecotono ChacoChiquitanía. Revista Boliviana de Ecología y Conservación Ambiental, 11, 55-65.

Maffei, L., Polisar, J., Garcia, R., Moreira, J. \& Noss, A. (2011). Perspectives from ten years of jaguar (Panthera onca) camera trapping in Mesoamerica. Mesoamericana, 15, 49-59 pp.

Martínez, Y. (2013). Perfil técnico del Corredor Biológico el Quetzal Tres Colinas. Centro Agronómico Tropical de Investigación $\quad y \quad$ Enseñanza. https://www.academia.edu/9353326/Perfil_T\%C3\%A9cnico_Corredor_Biol\%C3\%B3gico_Quetzal_Tres_Colinas

Maxwell, C. (2018). The allure of lure and its impact on perceived community composition when monitoring tropical mammalian biodiversity [Master's Thesis. University of Delaware].

McDonough, C., \& Loughry, W. (1997). Influences on activity patterns in a population of nine-banded armadillos. Journal of Mammalogy, 78, 932-941. https://doi.org/10.2307/1382953

Mech, L., \& Cluff, H. (2011). Movements of wolves at the northern extreme of the species range, including during four months of darkness. PLOS ONE. 6: 1-5. https://doi.org/10.1371/journal.pone.0025328

Meek, P., Ballard, G., Fleming, P., \& Falzon, G. (2016). Are we getting the full picture? Animal responses to camera traps and implications for predator studies. Ecology and Evolution, 6, 3216-3225 https://doi.org/10.1002/ece3.2111

Meek, P., Ballard, G., Fleming, P., Schaefer, M., Williams, W., \& Falzon, G. (2014). Camera traps can be heard and seen by animals. PLoS ONE, 9(10), 1-16. https://doi.org/iournal.pone.0110832

Mejía, A. (2014). Permeabilidad de la zona de amortiguamiento del Parque Internacional de la Amistad sobre la comunidad de mamíferos terrestres medianos y grandes [Tesis de maestría. Instituto de Ecología de México].

Meredith, M., \& Ridout, M. (2018a). Overlap: Estimates of coefficient of overlapping for animal activity patterns. (version 0.3.2)[software]. R package. https://cran.rproject.org/web/packages/overlap/overlap.pdf

Meredith, M., \& Ridout, M. (2018b). Overview of the overlap package. R package. https://cran.rproject.org/web/packages/overlap/vignettes/overlap.pdf

Michalski, F., \& Norris, D. (2011). Activity pattern of Cuniculus paca (Rodentia: Cuniculidae) in relation to lunar illumination and other abiotic variables in the southern Brazilian Amazon. Zoología, 28, 701-708. https://doi.org/10.1590/S1984-46702011000600002

Monroy, O., Zarco, M., Rodriguez, C., Soria, L., \& Urios, V. (2011). Fototrampeo de mamíferos en la Sierra Nanchititla, México. Revista de Biología Tropical, 59 (1), 373-383. https://doi.org/10.15517/rbt.v59i1.3206

Monsalve, S.; Mattar, S., \& González, M. (2009). Zoonosis transmitidas por animales silvestres y su impacto en las enfermedades emergentes y reemergentes. Medicina Veterinaria y Zootecnia, 14(2), 1762-1773. https://doi.org/10.21897/rmvz.361 
Mooring, M.; Eppert, A., \& Botts, R. (2020). Natural Selection of Melanism in Costa Rican Jaguar and Oncilla: A Test of Gloger's Rule and the Temporal Segregation Hypothesis. Tropical Conservation Science, 13: 1-15. https://doi.org/10.1177/1940082920910364

Mora, J. (2000). Los mamíferos silvestres de Costa Rica. Editorial Universidad Estatal a Distancia.

Moreno, R. (2000). Atrayentes para los felinos silvestres. Scientia, 15(1), 115-117.

Moreno, R.; Kays, R.; Giacalone, J.; Aliaga, E; Mares, R., \& Bustamante, A. (2012). Ámbito de hogar y actividad circadiana del Ocelote (Leopardus pardalis) en la Isla de Barro Colorado, Panamá. Mesoamericana, 16 (3), 30-39.

Morgan, H., Ballard, G., Fleming, P., Reid, N., Ven, R., \& Vernes, K. (2018). Estimating macropod grazing density and defining activity patterns using camera-trap image analysis. Wildlife Research. Vol. 45(8): $706-717 \mathrm{pp}$. https://doi.org/10.1071/WR17162

Negroes, N., Sarmento, P., Cruz, J., Eira, C., Revilla, E., Fonseca, C., Sollmann, R., Torres, N., Furtado, M., Jacomo, A., \& Silveira, L. (2010). Use of camera-trapping to estimate puma density and influencing factors in central Brazil. Journal of Wildlife Management, 74(6), 1195-1203. https://doi.org/10.1111/j.1937-2817.2010.tb01240.x

Norris, D., Michalski, F., \& Pérez, C. (2010). Habitat patch size modulates terrestrial mammal activity patterns in Amazonian forest fragments. Journal of Mammalogy, 91, 551-560. https://doi.org/10.1644/09-MAMM-A-199.1

Noss, A., Polisar, J., Maffei, L., Garcia, R., \& Silver, S. (2013). Evaluating jaguar densities with camera traps. Jaguar Conservation Program and Latin America and Caribbean Program Wildlife Conservation Society. https://bit.ly/3o5luM3

Nouvellet, P., Rasmussen, G., Macdonald, D., \& Courchamp, F. (2012). Noisy clocks and silent sunrises: Measurement methods of daily activity pattern. Journal of Zoology, 286, 179-184. https://doi.org/10.1111/j.14697998.2011.00864.x

O'Brien, T., Kinnaird, M., \& Wisibono, H. (2003). Crouching tigers, hidden prey: sumatran tiger and prey populations in a tropical forest landscape. Animal Conservation, 6 131-139 https://doi.org/10.1017/S1367943003003172

O'Connell, A., Nichols, J., \& Karanth, U. (2011). Camera traps in animal ecology: methods and analyses. Springer. https://doi.org/10.1007/978-4-431-99495-4

Oksanen, J., Blanchet, F.G., Friendly, M., Kindt, R., Legendre, P., McGlinn, D., Minchin, P.R., O'Hara, R.B., Simpson, G.L., Solymos, P., Stevens, H.M., Szoecs, E., and Wagner H. (2019). Package vegan (version 2.5-6) [software] Community Ecology Package. R package https://CRAN.R-project.org/package=vegan

Oliveira, L., Machado, L., Tortato, M., \& Brusius, L. (2010). Influence of extrinsic variables on activity and habitat selection of lowland tapirs (Tapirus terrestris) in the coastal sand plain shrub, southern Brazil. Mammalian Biology, 75, 219226. https://doi.org/10.1016/j.mambio.2009.05.006

Ortiz, E. (2014). Atlas digital de Costa Rica. (versión 2014) [software]. Instituto Tecnológico de Costa Rica. https://repositoriotec.tec.ac.cr/handle/2238/6749

Owen, N. (1998). How high ambient temperature affects the daily activity and foraging time of a subtropical ungulate, the greater kudu (Tragelaphus strepsiceros). Journal of Zoology, 246, 183-192. https://doi.org/10.1111/j.14697998.1998.tb00147.x

Palminteri, S., Powell, G., Fernandéz, A., \& Tovar, D. (1999). Talamanca Montane-Isthmian Pacific ecoregion-based conservation plan: preliminary reconnaissance phase. Tropical Science Center, Costa Rica.

Parsons, A., Forrester, T., McShea, W., Whatton, M., Millspaugh, J., \& Kays R. (2017). Do occupancy or detection rates from camera traps reflect deer density? Journal of Mammalogy, 98(6), 1547-1557. https://doi.org/10.1093/jmammal/gyx128 
Penteriani, V., Kuparinen, A., Delgado, M., Palomares, F., López, J., \& Fedriani, J. (2013). Responses of a top, a meso predator and their prey to moon phases. Oecologia, 173, 753-766. https://doi.org/10.1007/s00442-013-2651-6

Piedra, L., \& Maffei, L. (2000). Efecto de las actividades humanas sobre la diversidad de mamíferos terrestres en un gradiente altitudinal. Revista de Biología Tropical, 48(1), 263-264

PNUMA-CMCM. (2014). Lista de especies CITES. Secretaría CITES, Ginebra, Suiza, y PNUMACMCM, Cambridge, Reino Unido. Recuperado el 2 de mayo del 2021. http://checklist.cites.org/\#/es

Polisar, J., O’Brien, T., Matthews, S., Beckmann, J., Sanderson, E., Rosas, O., \& López, C. (2014). Review of Jaguar Survey and monitoring techniques and methodologies. (Wildlife Conservation Society final report) U.S. Fish and Wildlife Service.

https://www.fws.gov/southwest/es/arizona/Documents/SpeciesDocs/Jaguar/Polisar\%20et\%20al\%202014\%20R eview\%20Final.pdf

Prugh, L., \& Golden, C. (2014). Does moonlight increase predation risk? Meta-analysis reveals divergent responses of nocturnal mammals to lunar cycles. Journal of Animal Ecology, 83, 504-514. https://doi.org/10.1111/13652656.12148

QGIS Development Team. (2020). QGIS geographic information system (versión 3.10) [software]. Open Source Geospatial Foundation Project. http://agis.osgeo.org

R Core Team (2019). R: A language and environment for statistical computing. (version 4.1.0) [software]. R Foundation for Statistical Computing. https://www.R-project.org

Ramesh, T., Kalle, R., Sankar, K., \& Qureshi, Q. (2012). Spatio-temporal partitioning among large carnivores in relation to major prey species in Western Ghats. Journal of Zoology, 287(4), 269-275. https://doi.org/10.1111/j.14697998.2012.00908.x

Ramos, I., Guerrero, S., \& Huerta, F. (2007). Patrones de distribución geográfica de los mamíferos de Jalisco, México. Revista Mexicana de Biodiversidad, 78, 175-189. https://doi.org/10.22201/ib.20078706e.2007.001.392

Randel, C., \& Peace, D. (2010). Coyote behavioral response to scent-stations. Transactions of Western Section of the Wildlife Society, 45, 17-20.

Ridout, M., \& Linkie, M. (2009). Estimating overlap of daily activity patterns from camera trap data. Journal of Agricultural, Biological and Environmental Statistics, 14(3), 322-337. https://doi.org/10.1198/iabes.2009.08038

Rodríguez, B., Chinchilla, F., \& May, L. (2002). Lista de especies, endemismo y conservación de los mamíferos de Costa Rica. Revista Mexicana de Mastozoología. 6: 21-57. https://doi.org10.22201/ie.20074484e.2002.6.1.104

Rodríguez, B., Ramírez, J., Villalobos, D., \& Sánchez, R. (2014). Actualización de la lista de especies de mamíferos vivientes de Costa Rica. Mastozoología Neotropical, 21(2), 275-289.

Ross, J., Hearn, A., Johnson, P., \& Macdonald, D. (2013). Activity patterns and temporal avoidance by prey in response to Sunda clouded leopard predation risk. Journal of Zoology, 290(2), 96-106. https://doi.org/10.1111/jzo.12018

Rovero, F., \& Marshall, A. (2009). Camera trapping photographic rate as an index of density in forest ungulates: methodological insights. Journal of Applied Ecology, 46, 1011-1017. https://doi.org/10.1111/j.1365$\underline{2664.2009 .01705 . x}$

Rovero, F., Martin, E., Rosa, M., Ahumada, J.A., Spitale, D. (2014) Estimating Species Richness and Modelling Habitat Preferences of Tropical Forest Mammals from Camera Trap Data. PLoS ONE 9(7): http://doi.org/10.1371/journal.pone.0103300

Rowcliffe, J. M., Kays, R., Kranstauber, B., Carbone, C., \& Jansen, P. A. (2014). Quantifying levels of animal activity using camera trap data. Methods in Ecology and Evolution, 5(11), 1170-1179. https://doi.org/:10.1111/2041$\underline{210 X .12278}$ 
Russo, D., Maglio, G., Rainho, A., Meyer, C., \& Palmeirim, J. (2011). Out of the dark: diurnal activity in the bat Hipposideros ruber on São Tomé Island (West Africa). Mammalian Biology, 76, 701-708. https://doi.org/10.1016/i.mambio.2010.11.007

Sáenz, J. \& Carrillo, E. (2002). Chancho de monte en la Península de Osa. Ecología e importancia. Ambientales, 24, 1-10. https://doi.org/10.15359/rca.24-1.1

Salom, R., Polisar, J., Quigley, H., \& Zeller, K. (2010). Iniciativa del Corredor del Jaguar: un corredor biológico y un compromiso a largo plazo para la conservación. Mesoamericana, 14 (3), 25-34 pp.

Sánchez, R., Brenes, L., Chavarría, K., \& Mejías, Y. (2019). Diversidad y patrones de actividad de mamíferos medianos y grandes, en el sendero La Fila, Reserva Biológica Alberto Manuel Brenes, Alajuela, Costa Rica. Pensamiento Actual, 19(33), 175-189. https://doi.org/10.15517/pa.v19133.39619

Sanderson, J., \& Harris, G. (2013). Automatic data organization, storage, and analysis of camera trap pictures. Journal of Indonesian Natural History, 1(1), 1-9.

Sanderson, J., \& Trolle, M. (2005). Monitoring elusive mammals. American Scientist, 93, 148-155. https://doi.org/10.1511/2005.2.148

Santos, A., Velásquez, E., \& Sánchez, A. (2010). Effect of the intensity of the moonlight and wind speed in the activity of phyllostomid bats of Mena Nizanda, Oaxaca, Mexico. Revista Mexicana de Biodiversidad, 81, 839-845. https://doi.org/10.22201/ib.20078706e.2010.003.653

Séquin, E., Jaeger, M., Brussard, P., \& Barrett, R. (2003). Wariness of coyotes to camera traps relative to social status and territory boundaries. Canadian Journal of Zoology, 81, 2015-2025. https://doi.org/10.1139/z03-204

$\mathrm{Si}$, X., Kays, R., \& Ding, P. (2014). How long is enough to detect terrestrial animals? Estimating the minimum trapping effort on camera traps. PeerJ 2, e374. 1-14. https://doi.org/10.7717/peerj.374

Silveira, L., Jácomo, A., \& Diniz, J. (2003). Camera trap, line transect census and track surveys: a comparative evaluation. Biological Conservation, 114, 351-355. https://doi.org/10.1016/S0006-3207(03)00063-6

SINAC. (2012). Plan General de manejo Parque Internacional de La Amistad. (2012-2019). Área de Conservación La Amistad Pacífico y Área de Conservación La Amistad Caribe. https://bit.ly/2Y5g5MW

SINAC. (2017). Listado de flora y fauna en peligro de extinción y con poblaciones reducidas o amenazadas. Resolución RSINAC-CONAC-092-2017. Sistema Costarricense de información Jurídica. Recuperado el 15 de mayo de 2021. https://bit.ly/3BE7cHQ

SINAC. (2019). Plan General de Manejo del Parque Internacional de La Amistad. (2020-2029). Área de Conservación La Amistad Pacífico y Área de Conservación La Amistad Caribe. https://bit.ly/3GK2CLS

Sollmann, R. (2018). A gentle introduction to camera-trap data analysis. African Journal of Ecology, 56, $740-749$. https://doi.org/10.1111/aje.12557

Soria, L., \& Monroy, O. (2015). Monitoring population density and activity pattern of white-tailed deer (Odocoileus virginianus) in Central Mexico, using camera trapping. Mammalia, 79(1), 43-50. https://doi.org/10.1515/mammalia-2013-0107

Srbek, A., Haag, T., Chiarello, A., Salzano, F. \& Eizirik, E. (2018). Worrisome isolation: Noninvasive genetic analyses shed light on the critical status of a remnant jaguar population. Journal of Mammalogy, 99, 397-407. https://doi.org/10.1093/imammal/gyy007

Sridhari, H., Shankar, T., \& Mudappa, D. (2008). Mammal persistence and abundance in tropical rainforest remnants in the southern Western Ghads, India. Current Science, 94, 748-757. 
Suselbeek, L., JamEmsens, W., Hirsch, B., Kays, R., Rowcliffe, J., Zamora, V., \& Jansen, P. (2014). Food acquisition and predator avoidance in a Neotropical rodent. Animal Behaviour, 88, 41-48. https://doi.org/10.1016/j.anbehav.2013.11.012

Tan, W., Hamzah, N., Saaban, S., Zawakhir, N., Rao, Y., Jamaluddin, N., Cheong, F., Khalid, N., Mohdsaat, N., Zaidee, E., Hamdan, A., Chow, M., Low, C., Voon, M., Liang, S., Tyson, M., \& Gumal, M. (2018). Observations of occurrence and daily activity patterns of ungulates in the Endau Rompin Landscape, peninsular Malaysia. Journal of Threatened Taxa, 10(2), 11245-11253 pp. https://doi.org/10.11609/jott.3519

Thorn, M., Scott, D., Green, M., Bateman, P., \& Cameron, E. (2009). Estimating brown hyaena occupancy using baited camera traps. African Journal of Wildlife Research, 39(1), 1-10. https://doi.org/10.3957/056.039.0101

Tiku, M. (1965). Chi-square approximations for the distributions of goodness-of-fit statistics $U^{2}$ and $W_{2}$. Biometrika, 52, 630-633. https://doi.org/10.2307/2333714

Tobler, M., Carrillo, S., Leite, R., Mares, R., \& Powell, G. (2008). An evaluation of camera traps for inventorying largeand medium-sized rainforest mammals. Animal Conservation, 11,169-178. https://doi.org/10.1111/j.1469$\underline{1795.2008 .00169 . x}$

Tobler, M., Naranjo, E., \& Lira, I. (2006). Habitat preference, feeding habits and conservation of Baird's tapir in Neotropical montane oak forests. Ecology and Conservation of Neotropical Montane Oak Forests. Ecological Studies, 185, 347359. https://doi.org/10.1007/3-540-28909-7 27

Trejo, J., Rebolledo, G., \& Rau, J. (2010). Uso y selección de hábitat por mamíferos carnívoros y herbívoros en bosque nativo y plantaciones forestales del sur de Chile. Gestión Ambiental, 19, 33-46.

UICN, (2012). Categorías y criterios de la Lista Roja de la UICN (Versión 3.1). Unión Internacional para la Conservación de La Naturaleza. https://portals.iucn.org/library/sites/library/files/documents/RL-2001-001-2nd-Es.pdf

UICN, (2020). The UICN red list of threatened species. The UICN Red List of threatened species. Version 2020-1. Recuperado el 15 de marzo del 2021 de https://www.iucnredlist.org

Upton, G. (1992). Fisher's exact test. Royal Statistical Society, 155(3), 95-402. https://doi.org/10.2307/2982890

Van Berkel, T. (2014). Camera trapping for wildlife conservation: Expedition field techniques. Royal Geographical Society. https://bit.ly/3wdKJR2

Van Schaik, C., \& Griffiths, M. (1996). Activity periods of Indonesian rain forest mammals. Biotropica, 28(1), $105-112$. https://doi.org/10.2307/2388775

Vásquez, L., \& Matallana, C. (2016). ¿Responden las investigaciones en las Áreas Protegidas de Risaralda a las necesidades de manejo y gestión de la biodiversidad? Ambiente y Desarrollo, 20 (38), 27-40. https://doi.org/10.11144/Javeriana.ayd20-38.riap

Vila, A., Aprile, G., Sotelo, V., Sugliano, P., Zoratti, C., Berardi, M., \& Montbrun. J. (2016). Cámaras trampa y huemules: ¿Una alternativa de monitoreo? Anales Instituto Patagonia, 44 (3), 71-76. http://dx.doi.org/10.4067/S0718$\underline{686 \times 2016000300007}$

Villate, R., \& Canet, L. (2010). Corredores Biológicos, su importancia para la gestión de paisajes marinos. Mesoamericana, 14(3), 105-113.

Villate, R., Canet, L., Chassot, O., \& Monge, G. (2010). Corredor Biológico San Juan-La Selva, Costa Rica: Lecciones Aprendidas de La Gestión de un Paisaje Funcional. Mesoamericana, 14(3), 88-94.

Wainwright, M. (2007). The mammals of Costa Rica. Zona Tropical Publication, Cornell University Press.

Walker, S., Novaro, A., \& Nichols, J. (2000). Consideraciones para la estimación de abundancia de poblaciones de mamíferos. Mastozoología Neotropical, 7, 73-80. 
Weaver, J., Wood, P., Paetkau, D., \& Laack, L. (2005). Use of scented hair snares to detect ocelots. Wildlife Society Bulletin, 33(4), 1384-1391. http://dx.doi.org/10.2193/0091-7648(2005)33[1384:UOSHST]2.0.CO;2

Williams, S., Marsh, H., \& Winter, J. (2002). Spatial scale, species diversity and habitat structure: small mammals in Australian tropical rain forest. Ecology, 83(5), 1317-1329. http://dx.doi.org/10.2307/3071946

Wright, J., Byers, J., Koukoumaftsis, L., Ralph, P., \& Gribben, P. (2010). Native species behavior mitigates the impact of habitat-forming invasive seaweed, Oecologia, 163 (2), 527-534. http://dx.doi.org/10.1007/s00442-010-1608-2

Young, S., Rode-Margono, J., \& Amin, R. (2018). Software to facilitate and streamline camera trap data management: A review. Ecology and Evolution, 8, 9947-9957. http://dx.doi.org/10.1002/ece3.4464

Zar, J. (1999). Biostatistical analysis. Prentice Hall. 461-470 pp.

Zúñiga, A., \& Jiménez, J. (2010). Uso de técnicas no invasivas en el estudio ecológico de carnívoros: un análisis cuantitativo. Gestión Ambiental, 19, 67-96. 


\section{APÉNDICE DIGITAL}

TABLA 1

\begin{tabular}{|c|c|c|c|c|c|c|c|}
\hline Piso altitudinal & $\begin{array}{l}\text { Zona de } \\
\text { vida }\end{array}$ & Conservación & $\begin{array}{c}\text { Estación de } \\
\text { fototrampeo }\end{array}$ & $\begin{array}{c}\text { Elevación } \\
\text { (msnm) }\end{array}$ & $\begin{array}{l}\text { Coordenadas } \\
\text { CRTM05 E }\end{array}$ & $\begin{array}{l}\text { Coordenadas } \\
\text { CRTM05 N }\end{array}$ & Nombre común del sitio \\
\hline Premontano & $\mathrm{BmH}-\mathrm{P}$ & CBQTC & 1 & 920 & 00600212 & 01003135 & Finca helechales \\
\hline Premontano & $\mathrm{BmH}-\mathrm{P}$ & CBQTC & 2 & 1145 & 00601707 & 01003950 & Carril finca \\
\hline Premontano & BmHP-TP & CBQTC & 3 & 1438 & 00603154 & 01005298 & Finca Matamoros \\
\hline Premontano & BmHP-TP & CBQTC & 4 & 1482 & 00602708 & 01005150 & Carril finca \\
\hline Premontano & BP-P & CBQTC & 5 & 1652 & 00603513 & 01007995 & El Bajo \\
\hline Premontano & BP-P & CBQTC & 6 & 1675 & 00602569 & 01006534 & Toma de agua \\
\hline Montano Bajo & BP-MB & CBQTC & 7 & 1726 & 00602386 & 01007843 & Guardianes Bosque \\
\hline Montano Bajo & BP-MB & CBQTC & 8 & 1971 & 00603433 & 01009215 & Línea cortafuego \\
\hline Montano Bajo & BP-MB & PILA & 9 & 2199 & 00602498 & 01010532 & Cerro Kutsi \\
\hline Montano Bajo & BP-MB & PILA & 10 & 2450 & 00602731 & 01012555 & Palo de tigre \\
\hline Montano & BP-M & PILA & 11 & 2700 & 00603350 & 01013860 & Laguna seca \\
\hline Montano & BP-M & PILA & 12 & 2768 & 00602958 & 01015986 & Campamento 1 \\
\hline Montano & BP-M & PILA & 13 & 2977 & 00603463 & 01017395 & Bosque nuboso \\
\hline Montano & BP-M & PILA & 14 & 2840 & 00601496 & 01020400 & Campamento 2 \\
\hline Montano & BP-M & PILA & 15 & 2967 & 00601828 & 01022228 & La Turbera \\
\hline Montano & BP-M & PILA & 16 & 3064 & 00603677 & 01023191 & El Páramo \\
\hline \multirow[t]{2}{*}{ Subalpino } & BP-S & PILA & 17 & 3205 & 00604332 & 01023659 & Cerro Apri \\
\hline & BP-S & PILA & 18 & 3503 & 00605594 & 01024453 & Cerro Kamuk \\
\hline
\end{tabular}

BmH-P: Bosque Muy húmedo Premontano. BmHP-TP: Bosque Muy húmedo Premontano Transición a Pluvial. BP-P: Bosque Pluvial Premontano. BP-MB: Bosque Pluvial Montano Bajo. BP-M: Bosque Pluvial Montano. BP-S: Bosque Pluvial Subalpino. CBQTC: Corredor Biológico el Quetzal Tres Colinas. PILA: Parque Internacional de La Amistad. CRTM: Costa

Rica Transversal Mercator 


\section{TABLA 2}

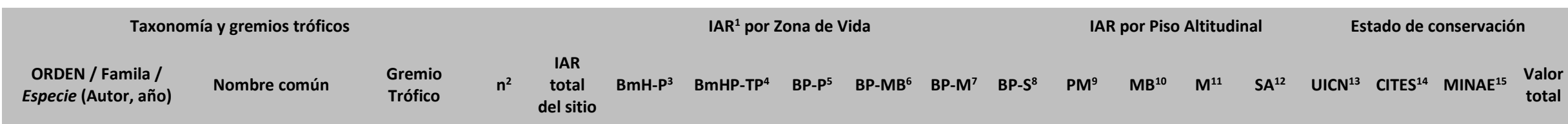

\section{CARNIVORA}

Felidae

Leopardus wiedii

(Schinz, 1821)

Leopardus tigrinus

(Schreber, 1775)

Leopardus pardalis

(Linnaeus, 1758)

Herpailurus

yagouaroundi

(É.Geoffroy Saint-

Hilaire, 1803)

Puma concolor

(Linnaeus, 1758)

Panthera onca

(Linnaeus, 1758)

Mustelidae

Mustela frenata

(Lichtenstein, 1831)

Eira barbara

(Linnaeus, 1758)

Procyonidae

Nasua narica

(Linnaeus, 1766)

Bassariscus sumichrasti

(Saussure, 1860)

Canidae

Canis latrans

(Say, 1823)

Mephitidae

Conepatus semistriatus

(Boddaert, 1785)

RODENTIA

Sciuridae

Sciurus granatensis

(Humboldt, 1811)

\begin{tabular}{|c|c|c|c|c|c|c|c|c|c|c|c|c|c|c|c|c|c|}
\hline Caucel, margay & Carnívoro & 35 & 2,54 & 3,77 & 8,21 & 7,25 & 3,84 & 0 & 3,09 & 0,35 & 4,15 & 2,57 & 3,09 & $\mathrm{CA}$ & । & $\mathrm{PE}$ & 8 \\
\hline Oncilla, tigrillo & Carnívoro & 81 & 5,88 & 0 & 0 & 0,91 & 4,15 & 2,57 & 6,17 & 0,35 & 0,64 & 11,32 & 6,17 & Vu & 1 & $\mathrm{PE}$ & 9 \\
\hline $\begin{array}{l}\text { Ocelote, } \\
\text { manigordo }\end{array}$ & Carnívoro & 31 & 2,25 & 0 & 0 & 0,91 & 0,64 & 11,32 & 6,17 & 6,62 & 3,84 & 0 & 0 & PM & 1 & $\mathrm{PE}$ & 7 \\
\hline León breñero & Carnívoro & 5 & 0,36 & 1,26 & 0 & 0,91 & 0,96 & 0 & 0 & 0,70 & 0,96 & 0 & 0 & PM & 1 & $\mathrm{PE}$ & 7 \\
\hline Puma o león & Carnívoro & 147 & 10,67 & 0 & 11,29 & 2,72 & 11,51 & 12,47 & 2,06 & 4,87 & 11,51 & 15,95 & 2,06 & PM & I & $\mathrm{PE}$ & 7 \\
\hline Jaguar, pantera & Carnívoro & 157 & 11,40 & 0 & 3,08 & 3,63 & 27,80 & 8,10 & 3,09 & 2,44 & 27,80 & 9,77 & 3,09 & $\mathrm{CA}$ & 1 & PE & 8 \\
\hline Comadreja & Carnívoro & 9 & 0,65 & 0 & 0 & 3,63 & 0,32 & 0,51 & 0 & 1,39 & 0,32 & 0,69 & 0 & PM & & & 2 \\
\hline Tolomuco, tayra & Omnívoro & 40 & 2,90 & 2,52 & 10,27 & 5,44 & 2,88 & 1,67 & 0,00 & 6,27 & 2,88 & 2,23 & 0 & PM & & & 2 \\
\hline Pizote & Omnívoro & 97 & 7,04 & 11,32 & 22,59 & 19,04 & 9,27 & 2,40 & 1,03 & 18,11 & 9,27 & 2,40 & 1,03 & PM & & & 2 \\
\hline Cacomiztle & Omnívoro & 23 & 1,67 & 0 & 0 & 0 & 0,96 & 2,57 & 0 & 0 & 0,96 & 3,43 & 0 & PM & & $\mathrm{PE}$ & 3 \\
\hline Coyote & Carnívoro & 12 & 0,87 & 0 & 1,03 & 0 & 2,88 & 0,26 & 0 & 0,35 & 2,88 & 0,34 & 0 & PM & & & 2 \\
\hline Zorrillo hediondo & Insectívoro & 47 & 3,41 & 3,77 & 3,08 & 9,07 & 6,39 & 1,41 & 0 & 5,57 & 6,39 & 1,89 & 0 & PM & & & 2 \\
\hline Ardilla colirroja & Hervíboro & 1838 & 133,41 & 41,51 & 98,56 & 219,40 & 277,40 & 77,03 & 40,12 & 129,18 & 277,40 & 89,33 & 40,12 & PM & & & 2 \\
\hline
\end{tabular}


Microsciurus alfari (J.A.Allen, 1895)

Agoutidae

Dasyprocta punctata

(Gray, 1842)

Cuniculidae

Cuniculus paca

(Linnaeus, 1766)

Eretizontidae

Coendou mexicanus

(Kerr, 1792)

ARTIODACTYLA

Tayassuidae

Tayassu pecari

(Link, 1795)

Pecari tajacu

(Linnaeus, 1766)

Cervidae

Mazama temama

(Kerr, 1792)

PRIMATES

Atelidae

Ateles geoffroyi

(Kuhl, 1820)

Cebidae

Cebus imitator

(Thomas, 1903)

DIDELPHIMORPHIA

Didelphidae

Didelphis marsupialis

(Linnaeus, 1758)

Philander opossum

(Linnaeus, 1758)

LAGOMORPHA

Leporidae

Sylvilagus dicei

(Harris, 1932)

PERISSODACTYLA

Tapiridae

Tapirus bairdii

(Gill, 1865)

CINGULATA

Dasypodidae

Dasypus novemcinctus

(Linnaeus, 1758)

\section{Ardilla enana}

Cherenga o

guatusa

Tepezcuintle

Puercoespín

Chancho de monte

Zaíno

Cabro de monte

Mono

colorado/araña

Mono carablanca

Zorro pelón

Zorro cuatro ojos

Conejo de monte

Danta o tapi

Hervíboro

Frugívoro

Omnívoro

Herbívoro

Hervíboro

Omnívoro

15

5,03

4,11

21,76

29,72

47,80

16,43

18,13

0

0,13

0

$\begin{array}{lllll}11,14 & 29,72 & 0,17 & 0 & \text { PM }\end{array}$

PM

Armadillo o cusuco

Insectívoro

$98 \quad 7,11 \quad 74,21$

4,53

4,15

4,12

$4,12 \quad 26,81 \quad 4,15 \quad 0 \quad 4,12 \quad P M$ 
${ }^{1}$ IAR: Índice de Abundancia Relativa. ${ }^{2} \mathrm{n}$ : número de eventos independientes ${ }^{3} \mathrm{BmH}-\mathrm{P}$ : Bosque Muy húmedo Premontano. ${ }^{4} \mathrm{BmHP}-\mathrm{TP}$ : Bosque Muy húmedo Premontano Transición a Pluvial. ${ }^{5} \mathrm{BP}-\mathrm{P}$ : Bosque Pluvial Premontano ${ }^{6}$ BP-MB: Bosque Pluvial Montano Bajo. ${ }^{7}$ BP-M: Bosque Pluvial Montano. ${ }^{8}$ BP-S: Bosque Pluvial Subaplino ${ }^{9}$ PM: Pre-montano. ${ }^{10} \mathrm{MB}$ : Montano Bajo. ${ }^{11} \mathrm{M}$ : Montano. ${ }^{12}$ SA: Sub-Alpino. ${ }^{13}$ UICN: Unión Internacional para la Conservación de la Naturaleza: CA: Casi Amenazado, VU: Vulnerable, PM: Preocupación menor. DI: Datos insuficientes. EP: En Peligro. ${ }^{14}$ CITES: Convención sobre el Comercio Internacional de Especies Amenazadas de Fauna y Flora. ${ }^{15}$ MINAE: Ministerio de Ambiente, Energía y Mares de Costa Rica. PE: Peligro de extinción PR: Poblaciones Reducidas

TABLA 3

\begin{tabular}{lclc}
\hline Especie (s) detectada (s) & Día cámara trampa & Especie (s) detectada (s) & Día cámara trampa \\
\hline Sciurus granatensis y Tapirus bairdii & 4 & Eira barbara & 44 \\
Leopardus tigrina y Sylvilagus dicei & 5 & Agouti paca & 92 \\
Mazama temama & 7 & Bassariscus sumichrasti & 102 \\
Conepatus semistriatus & 8 & Herpailurus yaguaroundi & 114 \\
Dasypus novemincinctus y Tayassu tajacu & 9 & Canis latrans & 136 \\
Puma concolor & 12 & Didelphis marsupialis & 141 \\
Panthera onca & 14 & Leopardus pardalis & 181 \\
Nasua narica & 16 & Dasiprocta punctata & 220 \\
Leopardus wiedii & 17 & Philander opossum & 298 \\
Coendou mexicanus & 19 & Tayassu pecari & 332 \\
Eira barbara & 44 & Mustela frenata & 349 \\
Agouti paca & 92 & Microsciurus alfari & 736 \\
Bassariscus sumichrasti & 102 & Cebus capucinus & 807 \\
Herpailurus yaguaroundi & 114 & Ateles geoffroyi & 991 \\
\hline
\end{tabular}




\section{TABLA 4}

\begin{tabular}{|c|c|c|c|c|c|c|c|c|c|c|c|}
\hline Especies silvestres & N1 & Especie domésticas & N2 & $\begin{array}{c}\text { Overlap } \\
\text { Estimate }\end{array}$ & $\begin{array}{c}95 \% \\
\text { Lower }\end{array}$ & $\begin{array}{l}95 \% \\
\text { Upper }\end{array}$ & $\begin{array}{c}\text { Watson } \\
\mathbf{U}^{2}\end{array}$ & $\mathbf{P}-\mathrm{U}^{2}$ & $\mathbf{W}_{\mathrm{r}}$ & $\mathbf{P}-\mathbf{W}_{\mathrm{r}}$ & P Fisher \\
\hline Bassariscus sumichrasti & 23 & Equus caballus & 12 & 0,07 & $-0,02$ & 0,19 & 0,62 & 0,000 & 24,11 & 0,000 & 0,001 \\
\hline Bassariscus sumichrasti & 23 & Homo sapiens & 1234 & 0,19 & 0,08 & 0,30 & 1,64 & 0,000 & 46,23 & 0,000 & 0,000 \\
\hline Bassariscus sumichrasti & 23 & Canis familiaris & 65 & 0,21 & 0,09 & 0,35 & 1,16 & 0,000 & 43,98 & 0,000 & 0,000 \\
\hline Bassariscus sumichrasti & 23 & Bos taurus & 53 & 0,29 & 0,14 & 0,44 & 0,94 & 0,000 & 36,56 & 0,000 & 0,000 \\
\hline Canis latrans & 12 & Equus caballus & 12 & 0,27 & 0,06 & 0,48 & 0,51 & 0,000 & 20,98 & 0,000 & 0,027 \\
\hline Canis latrans & 12 & Canis familiaris & 65 & 0,35 & 0,15 & 0,53 & 0,44 & 0,000 & 13,95 & 0,000 & 0,000 \\
\hline Canis latrans & 12 & Homo sapiens & 1234 & 0,37 & 0,19 & 0,56 & 0,52 & 0,000 & 18,05 & 0,000 & 0,000 \\
\hline Canis latrans & 12 & Bos taurus & 53 & 0,41 & 0,24 & 0,60 & 0,30 & 0,005 & 11,82 & 0,000 & 0,001 \\
\hline Coendou mexicanus & 39 & Equus caballus & 12 & 0,01 & $-0,02$ & 0,08 & 0,77 & 0,000 & 27,45 & 0,000 & 0,000 \\
\hline Coendou mexicanus & 39 & Homo sapiens & 1234 & 0,12 & 0,07 & 0,18 & 2,96 & 0,000 & 81,53 & 0,000 & 0,000 \\
\hline Coendou mexicanus & 39 & Canis familiaris & 65 & 0,17 & 0,08 & 0,27 & 1,75 & 0,000 & 68,77 & 0,000 & 0,000 \\
\hline Coendou mexicanus & 39 & Bos taurus & 53 & 0,27 & 0,16 & 0,40 & 1,56 & 0,000 & 62,07 & 0,000 & 0,000 \\
\hline Conepatus semistriatus & 47 & Equus caballus & 12 & 0,04 & $-0,03$ & 0,12 & 0,80 & 0,000 & 27,48 & 0,000 & 0,000 \\
\hline Conepatus semistriatus & 47 & Homo sapiens & 1234 & 0,13 & 0,09 & 0,19 & 3,39 & 0,000 & 96,97 & 0,000 & 0,000 \\
\hline Conepatus semistriatus & 47 & Canis familiaris & 65 & 0,14 & 0,06 & 0,24 & 1,95 & 0,000 & 76,44 & 0,000 & 0,000 \\
\hline Conepatus semistriatus & 47 & Bos taurus & 53 & 0,23 & 0,13 & 0,34 & 1,45 & 0,000 & 55,28 & 0,000 & 0,000 \\
\hline Cuniculus paca & 280 & Equus caballus & 12 & 0,01 & $-0,02$ & 0,05 & 0,96 & 0,000 & 26,75 & 0,000 & 0,000 \\
\hline Cuniculus paca & 280 & Homo sapiens & 1234 & 0,08 & 0,06 & 0,10 & 17,87 & 0,000 & 595,17 & 0,000 & 0,000 \\
\hline Cuniculus paca & 280 & Canis familiaris & 65 & 0,11 & 0,05 & 0,18 & 3,79 & 0,000 & 120,97 & 0,000 & 0,000 \\
\hline Cuniculus paca & 280 & Bos taurus & 53 & 0,20 & 0,11 & 0,31 & 2,89 & 0,000 & 90,91 & 0,000 & 0,000 \\
\hline Dasyprocta punctata & 21 & Equus caballus & 12 & 0,50 & 0,28 & 0,71 & 0,29 & 0,006 & 11,24 & 0,000 & 0,207 \\
\hline Dasyprocta punctata & 21 & Homo sapiens & 1234 & 0,69 & 0,52 & 0,83 & 0,27 & 0,010 & 10,28 & 0,100 & 0,001 \\
\hline Dasyprocta punctata & 21 & Bos taurus & 53 & 0,73 & 0,56 & 0,88 & 0,08 & 0,431 & 3,73 & 0,700 & 0,251 \\
\hline Dasyprocta punctata & 21 & Canis familiaris & 65 & 0,77 & 0,60 & 0,91 & 0,16 & 0,086 & 7,48 & 0,000 & 0,267 \\
\hline Dasypus novemcinctus & 98 & Equus caballus & 12 & 0,05 & $-0,01$ & 0,13 & 0,87 & 0,000 & 27,32 & 0,000 & 0,000 \\
\hline Dasypus novemcinctus & 98 & Homo sapiens & 1234 & 0,16 & 0,11 & 0,21 & 6,47 & 0,000 & 191,99 & 0,000 & 0,000 \\
\hline
\end{tabular}




\begin{tabular}{|c|c|c|c|c|c|c|c|c|c|c|c|}
\hline Dasypus novemcinctus & 98 & Canis familiaris & 65 & 0,19 & 0,11 & 0,28 & 2,51 & 0,000 & 96,62 & 0,000 & 0,000 \\
\hline Dasypus novemcinctus & 98 & Bos taurus & 53 & 0,28 & 0,16 & 0,39 & 1,88 & 0,000 & 71,45 & 0,000 & 0,000 \\
\hline Didelphis marsupialis & 126 & Equus caballus & 12 & 0,01 & $-0,02$ & 0,07 & 0,91 & 0,000 & 27,46 & 0,000 & 0,000 \\
\hline Didelphis marsupialis & 126 & Homo sapiens & 1234 & 0,10 & 0,07 & 0,13 & 8,97 & 0,000 & 266,79 & 0,000 & 0,000 \\
\hline Didelphis marsupialis & 126 & Canis familiaris & 65 & 0,13 & 0,07 & 0,21 & 3,14 & 0,000 & 116,37 & 0,000 & 0,000 \\
\hline Didelphis marsupialis & 126 & Bos taurus & 53 & 0,22 & 0,12 & 0,32 & 2,54 & 0,000 & 92,65 & 0,000 & 0,000 \\
\hline Eira barbara & 40 & Equus caballus & 12 & 0,66 & 0,47 & 0,83 & 0,20 & 0,041 & 8,46 & 0,000 & 0,134 \\
\hline Eira barbara & 40 & Bos taurus & 53 & 0,68 & 0,54 & 0,82 & 0,40 & 0,001 & 16,69 & 0,000 & 0,020 \\
\hline Eira barbara & 40 & Homo sapiens & 1234 & 0,85 & 0,74 & 0,94 & 0,10 & 0,306 & 4,37 & 0,300 & 0,223 \\
\hline Eira barbara & 40 & Canis familiaris & 65 & 0,86 & 0,75 & 0,95 & 0,06 & 0,632 & 2,47 & 0,900 & 0,078 \\
\hline Leopardus pardalis & 31 & Equus caballus & 12 & 0,08 & $-0,01$ & 0,20 & 0,72 & 0,000 & 26,73 & 0,000 & 0,000 \\
\hline Leopardus pardalis & 31 & Homo sapiens & 1234 & 0,22 & 0,12 & 0,34 & 1,98 & 0,000 & 57,86 & 0,000 & 0,000 \\
\hline Leopardus pardalis & 31 & Canis familiaris & 65 & 0,24 & 0,11 & 0,37 & 1,16 & 0,000 & 43,95 & 0,000 & 0,000 \\
\hline Leopardus pardalis & 31 & Bos taurus & 53 & 0,32 & 0,18 & 0,48 & 0,87 & 0,000 & 33,07 & 0,000 & 0,000 \\
\hline Leopardus tigrinus & 81 & Equus caballus & 12 & 0,20 & 0,10 & 0,31 & 0,67 & 0,000 & 24,14 & 0,000 & 0,002 \\
\hline Leopardus tigrinus & 81 & Homo sapiens & 1234 & 0,36 & 0,28 & 0,45 & 3,50 & 0,000 & 103,35 & 0,000 & 0,000 \\
\hline Leopardus tigrinus & 81 & Canis familiaris & 65 & 0,39 & 0,29 & 0,51 & 1,40 & 0,000 & 55,52 & 0,000 & 0,000 \\
\hline Leopardus tigrinus & 81 & Bos taurus & 53 & 0,47 & 0,34 & 0,60 & 0,86 & 0,000 & 33,21 & 0,000 & 0,000 \\
\hline Leopardus wiedii & 35 & Equus caballus & 12 & 0,14 & 0,02 & 0,28 & 0,67 & 0,000 & 25,04 & 0,000 & 0,000 \\
\hline Leopardus wiedii & 35 & Homo sapiens & 1234 & 0,26 & 0,16 & 0,36 & 2,06 & 0,000 & 61,56 & 0,000 & 0,000 \\
\hline Leopardus wiedii & 35 & Canis familiaris & 65 & 0,27 & 0,15 & 0,41 & 1,31 & 0,000 & 51,01 & 0,000 & 0,000 \\
\hline Leopardus wiedii & 35 & Bos taurus & 53 & 0,37 & 0,23 & 0,51 & 0,96 & 0,000 & 37,24 & 0,000 & 0,000 \\
\hline Mazama temama & 288 & Equus caballus & 12 & 0,47 & 0,34 & 0,58 & 0,35 & 0,002 & 14,15 & 0,000 & 0,089 \\
\hline Mazama temama & 288 & Homo sapiens & 1234 & 0,66 & 0,60 & 0,71 & 2,84 & 0,000 & 87,59 & 0,000 & 0,000 \\
\hline Mazama temama & 288 & Bos taurus & 53 & 0,71 & 0,60 & 0,80 & 0,24 & 0,017 & 6,61 & 0,000 & 0,041 \\
\hline Mazama temama & 288 & Canis familiaris & 65 & 0,74 & 0,66 & 0,82 & 0,48 & 0,000 & 19,43 & 0,000 & 0,024 \\
\hline Nasua narica & 97 & Bos taurus & 53 & 0,67 & 0,54 & 0,80 & 0,60 & 0,000 & 24,31 & 0,000 & 0,000 \\
\hline Nasua narica & 97 & Equus caballus & 12 & 0,69 & 0,50 & 0,84 & 0,16 & 0,080 & 6,75 & 0,100 & 0,202 \\
\hline Nasua narica & 97 & Canis familiaris & 65 & 0,87 & 0,77 & 0,95 & 0,06 & 0,611 & 2,25 & 0,900 & 0,106 \\
\hline
\end{tabular}




\begin{tabular}{|c|c|c|c|c|c|c|c|c|c|c|c|}
\hline Nasua narica & 97 & Homo sapiens & 1234 & 0,90 & 0,82 & 0,96 & 0,11 & 0,239 & 4,05 & 0,100 & 0,103 \\
\hline Panthera onca & 157 & Equus caballus & 12 & 0,38 & 0,24 & 0,51 & 0,54 & 0,000 & 20,61 & 0,000 & 0,002 \\
\hline Panthera onca & 157 & Homo sapiens & 1234 & 0,57 & 0,50 & 0,64 & 3,19 & 0,000 & 108,62 & 0,000 & 0,000 \\
\hline Panthera onca & 157 & Canis familiaris & 65 & 0,65 & 0,55 & 0,74 & 0,87 & 0,000 & 34,85 & 0,000 & 0,000 \\
\hline Panthera onca & 157 & Bos taurus & 53 & 0,73 & 0,62 & 0,82 & 0,30 & 0,005 & 11,43 & 0,000 & 0,085 \\
\hline Pecari tajacu & 101 & Equus caballus & 12 & 0,56 & 0,36 & 0,71 & 0,30 & 0,005 & 12,28 & 0,000 & 0,010 \\
\hline Pecari tajacu & 101 & Bos taurus & 53 & 0,72 & 0,59 & 0,84 & 0,20 & 0,042 & 8,63 & 0,000 & 0,003 \\
\hline Pecari tajacu & 101 & Homo sapiens & 1234 & 0,73 & 0,65 & 0,81 & 0,80 & 0,000 & 31,08 & 0,000 & 0,000 \\
\hline Pecari tajacu & 101 & Canis familiaris & 65 & 0,78 & 0,67 & 0,87 & 0,26 & 0,011 & 11,36 & 0,000 & 0,010 \\
\hline Philander opossum & 74 & Equus caballus & 12 & 0,01 & $-0,02$ & 0,07 & 0,86 & 0,000 & 28,10 & 0,000 & 0,000 \\
\hline Philander opossum & 74 & Homo sapiens & 1234 & 0,11 & 0,08 & 0,15 & 5,47 & 0,000 & 155,14 & 0,000 & 0,000 \\
\hline Philander opossum & 74 & Canis familiaris & 65 & 0,14 & 0,06 & 0,22 & 2,59 & 0,000 & 102,38 & 0,000 & 0,000 \\
\hline Philander opossum & 74 & Bos taurus & 53 & 0,23 & 0,13 & 0,34 & 2,10 & 0,000 & 82,78 & 0,000 & 0,000 \\
\hline Puma concolor & 147 & Equus caballus & 12 & 0,43 & 0,28 & 0,56 & 0,45 & 0,000 & 17,73 & 0,000 & 0,224 \\
\hline Puma concolor & 147 & Homo sapiens & 1234 & 0,59 & 0,51 & 0,66 & 2,67 & 0,000 & 88,17 & 0,000 & 0,000 \\
\hline Puma concolor & 147 & Canis familiaris & 65 & 0,61 & 0,51 & 0,71 & 0,97 & 0,000 & 38,30 & 0,000 & 0,000 \\
\hline Puma concolor & 147 & Bos taurus & 53 & 0,63 & 0,51 & 0,73 & 0,52 & 0,000 & 16,63 & 0,000 & 0,000 \\
\hline Sciurus granatensis & 1838 & Equus caballus & 12 & 0,50 & 0,32 & 0,65 & 0,39 & 0,001 & 15,53 & 0,000 & 0,031 \\
\hline Sciurus granatensis & 1838 & Bos taurus & 53 & 0,60 & 0,49 & 0,71 & 0,53 & 0,000 & 18,11 & 0,000 & 0,000 \\
\hline Sciurus granatensis & 1838 & Homo sapiens & 1234 & 0,63 & 0,60 & 0,66 & 9,58 & 0,000 & 369,89 & 0,000 & 0,000 \\
\hline Sciurus granatensis & 1838 & Canis familiaris & 65 & 0,67 & 0,59 & 0,75 & 0,71 & 0,000 & 28,47 & 0,000 & 0,000 \\
\hline Sylvilagus dicei & 357 & Equus caballus & 12 & 0,05 & 0,00 & 0,12 & 0,94 & 0,000 & 26,55 & 0,000 & 0,000 \\
\hline Sylvilagus dicei & 357 & Homo sapiens & 1234 & 0,17 & 0,14 & 0,20 & 19,48 & 0,000 & 683,90 & 0,000 & 0,000 \\
\hline Sylvilagus dicei & 357 & Canis familiaris & 65 & 0,23 & 0,15 & 0,31 & 3,48 & 0,000 & 113,01 & 0,000 & 0,000 \\
\hline Sylvilagus dicei & 357 & Bos taurus & 53 & 0,35 & 0,25 & 0,45 & 2,40 & 0,000 & 81,56 & 0,000 & 0,000 \\
\hline Tapirus bairdii & 736 & Equus caballus & 12 & 0,18 & 0,09 & 0,28 & 0,81 & 0,000 & 25,33 & 0,000 & 0,000 \\
\hline Tapirus bairdii & 736 & Homo sapiens & 1234 & 0,33 & 0,30 & 0,36 & 23,70 & 0,000 & 906,17 & 0,000 & 0,000 \\
\hline Tapirus bairdii & 736 & Canis familiaris & 65 & 0,38 & 0,30 & 0,46 & 2,84 & 0,000 & 95,33 & 0,000 & 0,000 \\
\hline Tapirus bairdii & 736 & Bos taurus & 53 & 0,48 & 0,39 & 0,58 & 1,55 & 0,000 & 54,51 & 0,000 & 0,000 \\
\hline
\end{tabular}


TABLA 5

\begin{tabular}{|c|c|c|c|c|c|c|c|c|c|c|c|c|}
\hline Especie & Estación & N1 & Estación & N2 & $\begin{array}{l}\text { Overlap } \\
\text { Estimate }\end{array}$ & $\begin{array}{c}95 \% \\
\text { Lower }\end{array}$ & $\begin{array}{l}95 \% \\
\text { Upper }\end{array}$ & $\begin{array}{c}\text { Watson } \\
U^{2}\end{array}$ & $\mathrm{P}-\mathrm{U}^{2}$ & $\mathbf{W}_{\mathrm{r}}$ & $\mathbf{P}-\mathbf{W}_{\mathrm{r}}$ & P Fisher \\
\hline Canis latrans & Lluviosa & 0 & Seca & 12 & NA & NA & NA & NA & NA & NA & NA & NA \\
\hline Dasyprocta punctata & Lluviosa & 15 & Seca & 6 & NA & NA & NA & NA & NA & NA & NA & NA \\
\hline Cuniculus paca & Lluviosa & 99 & Seca & 181 & 0,94 & 0,86 & 0,99 & 0,031 & 0,937 & 2,242 & 0,9 & 0,830 \\
\hline Sciurus granatensis & Lluviosa & 916 & Seca & 922 & 0,89 & 0,86 & 0,93 & 0,784 & 0,000 & 24,497 & 0 & 0,000 \\
\hline Tapirus bairdii & Lluviosa & 378 & Seca & 358 & 0,89 & 0,84 & 0,94 & 0,166 & 0,077 & 4,864 & 0,1 & 0,155 \\
\hline Philander opossum & Lluviosa & 47 & Seca & 27 & 0,88 & 0,75 & 0,99 & 0,045 & 0,756 & 2,967 & 0,7 & 0,567 \\
\hline Mazama temama & Lluviosa & 134 & Seca & 154 & 0,88 & 0,81 & 0,94 & 0,054 & 0,649 & 2,657 & 0,9 & 0,592 \\
\hline Homo sapiens & Lluviosa & 163 & Seca & 1071 & 0,88 & 0,82 & 0,93 & 0,317 & 0,004 & 11,038 & 0 & 0,000 \\
\hline Sylvilagus dicei & Lluviosa & 182 & Seca & 175 & 0,88 & 0,81 & 0,93 & 0,085 & 0,369 & 2,267 & 0,9 & 0,226 \\
\hline Dasypus novemcinctus & Lluviosa & 64 & Seca & 34 & 0,85 & 0,72 & 0,95 & 0,034 & 0,891 & 2,333 & 0,8 & 0,390 \\
\hline Eira barbara & Lluviosa & 22 & Seca & 18 & 0,82 & 0,63 & 0,96 & 0,071 & 0,484 & 3,235 & 0,7 & 0,066 \\
\hline Coendou mexicanus & Lluviosa & 12 & Seca & 27 & 0,81 & 0,59 & 0,98 & 0,062 & 0,569 & 4,026 & 0,2 & 0,407 \\
\hline Nasua narica & Lluviosa & 35 & Seca & 62 & 0,80 & 0,65 & 0,93 & 0,123 & 0,179 & 6,442 & 0,2 & 0,652 \\
\hline Pecari tajacu & Lluviosa & 34 & Seca & 67 & 0,80 & 0,67 & 0,90 & 0,042 & 0,794 & 2,035 & 0,9 & 0,444 \\
\hline Leopardus tigrinus & Lluviosa & 48 & Seca & 33 & 0,79 & 0,64 & 0,91 & 0,060 & 0,588 & 2,733 & 0,7 & 0,647 \\
\hline Didelphis marsupialis & Lluviosa & 25 & Seca & 101 & 0,79 & 0,63 & 0,91 & 0,121 & 0,186 & 5,424 & 0,1 & 0,090 \\
\hline Puma concolor & Lluviosa & 56 & Seca & 91 & 0,76 & 0,64 & 0,88 & 0,220 & 0,026 & 9,019 & 0,1 & 0,156 \\
\hline Bassariscus sumichrasti & Lluviosa & 10 & Seca & 13 & 0,74 & 0,49 & 0,94 & 0,072 & 0,480 & 3,554 & 0,7 & 0,373 \\
\hline Conepatus semistriatus & Lluviosa & 16 & Seca & 31 & 0,73 & 0,54 & 0,90 & 0,088 & 0,349 & 4,468 & 0,6 & 0,522 \\
\hline Panthera onca & Lluviosa & 84 & Seca & 73 & 0,72 & 0,59 & 0,83 & 0,402 & 0,001 & 16,375 & 0 & 0,008 \\
\hline Leopardus wiedii & Lluviosa & 19 & Seca & 16 & 0,68 & 0,47 & 0,86 & 0,133 & 0,147 & 6,005 & 0 & 0,647 \\
\hline Leopardus pardalis & Lluviosa & 11 & Seca & 20 & 0,67 & 0,43 & 0,87 & 0,146 & 0,112 & 6,427 & 0 & 0,095 \\
\hline
\end{tabular}




\section{TABLA 6}

\begin{tabular}{ccc}
\hline Año & $\begin{array}{c}\text { Turistas en sendero Tres } \\
\text { Colinas - Cerro Kamuk PILA }\end{array}$ & Caminantes CBQTC \\
\hline 2018 & 34 & 9 \\
2019 & 97 & 47 \\
2020 & 79 & 75 \\
2021 & 91 & 83 \\
Total & 301 & 214 \\
\hline
\end{tabular}

